\title{
Asymptotic stability and transient growth in pulsatile Poiseuille flow through a compliant channel
}

\author{
Konstantinos Tsigklifis ${ }^{1} \dagger$, and Anthony D. Lucey ${ }^{1}$ \\ ${ }^{1}$ Fluid Dynamics Research Group, Department of Mechanical Engineering, Curtin University, \\ Western Australia 6845, Australia
}

(Received xx; revised xx; accepted xx)

The time-asymptotic linear stability of pulsatile flow in a channel with compliant walls is studied together with the evaluation of modal transient growth within the pulsation period of the basic flow as well as non-modal transient growth. Both one (verticaldisplacement) and two (vertical and axial) degrees-of-freedom compliant-wall models are implemented. Two approaches are developed to study the dynamics of the coupled fluidstructure system, the first being a Floquet analysis in which disturbances are decomposed into a product of exponential growth and sum of harmonics while the second is a timestepping technique of the evolution of the fundamental solution (monodromy) matrix. A parametric study of stability in the non-dimensional parameter space, principally defined by Reynolds number $(R e)$, Womersley number $(W o)$ and amplitude of the applied pressure modulation $(\Lambda)$, is then conducted for compliant walls of fixed geometric and material properties. The flow through a rigid channel is shown to be destabilised by pulsation for low $W_{o}$, stabilised due to Stokes-layer effects at intermediate $W_{o}$ while the critical Re approaches the steady Poiseuille-flow result at high $W o$ and that these effects are made more pronounced by increasing $\Lambda$. Wall flexibility is shown to be stabilising throughout the $W o$-range but, for the relatively stiff wall used, is more effective at high Wo. Axial displacements are shown to have negligible effect on the results based upon only vertical deformation of the compliant wall. The effect of structural damping in the compliant-wall dynamics is destabilising thereby suggesting that the dominant inflectional (Rayleigh) instability is of the Class A (negative-energy) type. It is shown that very high levels of modal transient growth can occur at low Wo and this mechanism could therefore be more important than asymptotic amplification in causing transition to turbulent flow for two-dimensional disturbances. Wall flexibility is shown to ameliorate mildly this phenomenon. As $W o$ is increased modal transient growth becomes progressively less important and the non-modal mechanism can cause similar levels of transient growth. We also show that oblique waves having non-zero transverse wave numbers are stable to higher values of critical $R e$ than their twodimensional counterparts. Finally, we identify an additional instability branch at high $R e$ that corresponds to wall-based travelling-wave flutter. We show that this is stabilised by the inclusion of structural damping, thereby confirming that it is of the Class $\mathrm{B}$ (positive-energy) instability type.

\section{Introduction}

Studies of the stability of a modulated unsteady base flow over a rigid wall date back a number of decades while in recent years attention has been given to the stability of steady

$\dagger$ Email address for correspondence: k.tsigklifis@curtin.edu.au 
base flow through a compliant channel. Potential applications of these fundamental systems range from the enhancement of heat-mass-momentum transfer through the destabilization of an otherwise stable flow through to skin-friction drag reduction if the modulation or the fluid structure interaction (FSI) has a stabilizing effect on the base flow. The present work combines these hitherto separate strands of investigation to analyse the stability of modulated flow through a compliant channel. Clearly, the present system has applications in both engineered and biomechanical systems, the latter in consideration of blood flow through small vessels wherein the unsteady base flow is laminar.

The earliest comprehensive stability study of a modulated base flow through a rigid channel is the Floquet analysis of Kerczek \& Davis (1974) and was summarised by Davis (1976). It was shown that the Stokes layer always has a stabilizing effect on the TollmienSchlichting waves (TSW) since a disturbance decays faster inside the Stokes layer than it would in a fluid with zero base flow wherein only viscous dissipation is rate-determining. In the latter paper it was shown that the most unstable part of the flow was outside of the Stokes layer. However, it was also recognised that the highly inflectional velocity profiles during parts of the base-flow cycle had a destabilizing effect but only when the modulation frequency was small so that growth within the cycle occurs on a sufficiently rapid scale to outweigh the time-varying change of the base state.

Later work by Kerczek (1982), also using a Floquet analysis, on the stability of plane pulsatile Poiseuille flow through rigid channels predicted that it is more stable than the corresponding steady plane Poiseuille flow for a range of imposed oscillation frequencies from $\omega_{c} / 10$ to $8 \omega_{c}$, where $\omega_{c}$ is the frequency of the critical mode for steady plane Poiseuille flow, and for the amplitude of velocity modulation (relative to the underlying steady flow) up to $\Lambda_{v}=0.25$. Outside of this range of frequencies, it was found that modulation was destabilizing as found in the numerical simulations of Singer et al. (1989) and the Floquet analysis of Hall (1975) for very high frequency modulation. In addition, Singer et al. (1989) found that the maximum stabilization is achieved when the ratio of the Stokes layer thickness to distance from the wall of the steady-flow critical layer is about 0.4 .

Both the Floquet analysis of Kerczek (1982) and the numerical simulations of Singer et al. (1989) revealed that for relatively low frequency modulation, there is a large increase of the disturbance kinetic energy during the part of the oscillatory cycle in which there are regions of inflection in the velocity profile and that this could trigger transition to turbulence by initiating nonlinear secondary instabilities, even if all the initial disturbances are in the linear range and the flow is linearly stable if integrated over a cycle. This finding was in agreement with the experimental observations of the stability of flow in the aorta (Nerem et al. 1972) and in a pipe (Einav \& Sokolov 1993) where it was concluded that the inflection of the velocity profile during the systolic deceleration is the most important factor for the observed instabilities. In contrast to the aforementioned studies, Straatman et al. (2002) conducted a Floquet analysis of plane pulsatile Poiseuille flow over rigid walls and found that the modulation is destabilizing for the entire range of frequency modulation. They also identified a critical Womersley number, representing the balance between viscous and inertial forces, at which modulation exercises the maximum level of destabilization.

We now turn attention to the stability of steady flow through compliant channels or over compliant walls (infinite channel height). Local linear stability analysis, assuming spatial growth, and numerical simulations of plane Poiseuille flow over finite compliant panels accounting only for vertical panel displacements were respectively presented by Davies \& Carpenter (1997a) and Davies \& Carpenter (1997b). Within this system, they verified the possible occurrence of the fluid-based unstable TSWs, flow-induced 
wall-based travelling-wave flutter (TWF) and divergence instability, and their modal interactions, that have been predicted for Blasius flow (Carpenter \& Morris 1990; Lucey \& Carpenter 1995) and potential flow (Lucey \& Carpenter 1992) over compliant walls. They also showed that even very short compliant panels are able to suppress the growth of TSWs and that the panel edges exhibit non-trivial singular behaviour causing waves to propagate upstream from the trailing edge with a wavelength close to that of the TSW or evanescent waves to exist as a result of the fixed leading-edge boundary condition.

Wall compliance can stabilize TSWs as a means of transition postponement when its structural dynamics (determined by geometric parameters and material properties) interact with the flow to generate a reduction to Reynolds-stress energy production integrated through the boundary layer (Carpenter \& Morris 1990). However, to postpone transition the compliant wall must not succumb to the wall-based instabilities that can provide an alternative route to transition as demonstrated by Lucey \& Carpenter (1995) when modelling the experiments of Gaster (1988). Carpenter \& Morris (1990), Davies \& Carpenter (1997a) and Carpenter \& Gajjar (1990) demonstrated that the viscous wall layer stabilizes TWF which is caused by the irreversible energy transfers from the flow to the wall via the existence of a critical layer that generates a phase difference between the wall velocity and perturbation fluid pressure. The effect of structural damping, that removes energy from the wall, is stabilizing for TWF. These studies also predict that divergence instability is stabilized by the critical layer and destabilized by the viscous wall layer. However, it has since been accepted that energy-dissipation effects have little to no bearing upon the critical flow speed at which divergence occurs because exactly at onset it is a static wave (Pitman \& Lucey 2009). Nevertheless, flow shear in the boundary layer does play a part in the determination of its critical flow speed as shown by Kapor et al. (2009).

In light of the foregoing overviews, the interaction of a modulated base flow with wall compliance has the potential to support a rich variety of system dynamics. One investigation of such a system has been conducted by Thaokar \& Kumaran (2004), using a Floquet analysis of modulated Couette flow over both a spring-backed flexible plate accounting for both vertical and axial structural displacements and an incompressible viscoelastic gel for low and intermediate Reynolds numbers. They found a purely oscillatory instability in the limit of zero Reynolds number which is stabilized by an increase in frequency. This instability occurs through the work done by the action of the viscous fluid stress on the tangential motion of the compliant wall. They then showed that the corresponding instability due to steady base flow (over a compliant wall) and the effect of base-flow oscillations reinforce each other, and were able to map the instability bounds using a non-dimensional parameter that represented the difference between the steady and oscillatory strain rates.

In the present work, we study the stability of the plane pulsatile Poiseuille flow over spring-backed compliant walls in order to assess the effect of the pressure modulation amplitude and its frequency on the stability of the FSI system. We implement compliant-wall models which allow both vertical and vertical-axial displacements. In order to validate the models, but also to study the transient stability of the FSI system, we implement two different numerical procedures for the Floquet analysis. In the first method, following Kerczek (1982); Schmid \& Henningson (2001); Thomas et al. (2011), the amplitude of the disturbances are expressed as the product of the temporal exponential growth and a sum of Fourier modes and a final generalized eigenvalue matrix problem is formulated. In the second method, a time-stepping procedure is developed, based on the temporal evolution of the fundamental solution matrix, the eigenvalues of which at the end of the fundamental period (monodromy matrix) define the system stability (Iooss \& 
Joseph 1990; Seydel 1988), as the number of periods of oscillation asymptotes to infinity. This time-stepping algorithm is used to study the modal transient amplification of the disturbances within the base-flow oscillatory cycle for a given spatial wavenumber, by finding the largest singular value of the fundamental matrix or the maximum value of the Rayleigh quotient for each time step (Meyer 2000; Schmid \& Henningson 2001). Alternatively, the resulting initial value problem is integrated in time with a random initial vector, monitoring the energy of the FSI system during the oscillation cycle (Kerczek 1982). Finally, the early non-modal transient amplification of the disturbances due to the non-normality of the Navier-Stokes operator, is studied by defining the energy norm of the fundamental matrix (Reddy et al. 1993; Schmid 2007; Hœpffner et al. 2010) which is calculated from the extracted eigenvalues and eigenvectors of the fundamental matrix at each time step of the response evolution.

Our analysis also considers three dimensional modes. Squire's theorem states that shear flows with rigid walls first become exponentially unstable to two-dimensional wave perturbations at a value of the Reynolds number that is smaller than any value for which unstable three-dimensional perturbations exist (Schmid \& Henningson 2001). However, this theorem cannot be applied for viscous flows bounded by compliant walls or for the transient growth of disturbances. It has been shown by Yeo (1992), when studying the linear stability of the Blasius boundary layer over compliant walls, that three-dimensional, or oblique, TSW modes tend to become more dominant than their two-dimensional counterparts for sufficiently compliant walls. Thus, the critical Reynolds number for the TSW neutral curves of a compliant wall is determined by three-dimensional modes. Accordingly, we investigate the effect of the three-dimensional disturbances on the timeasymptotic stability (as the number of periods asymptotes to infinity) and the modal transient growth (within the fundamental period) of plane pulsatile Poiseuille flow over relatively stiff isotropic compliant walls. This serves to show how the wavelength of spanwise disturbances affects the neutral-stability curves generated for two-dimensional disturbances in modulated plane Poiseuille flow as well as the maximum modal transient amplification of the disturbances.

The remainder of this paper is organized as follows. The formulation of the base and the three-dimensional disturbance fields for the flow over spring-backed compliant walls with only vertical displacements is described in Section 2 followed by Section 3 in which we describe the structural model for a compliant wall that admits both vertical and axial displacements. The methods of solution for the stability problem are described in Section 4. Results for the time-asymptotic stability problem, modal transient growth within the period of base-flow modulation and non-modal transient growth are presented and discussed in Section 5. Concluding remarks are given in Section 6.

\section{System Modelling}

\subsection{Base flow field}

We consider the plane pulsatile Poiseuille flow through two compliant walls separated by $2 L^{*}$ distance as illustrated by figure 1 . We consider as characteristic length scale, $L^{*}$, the half distance of the separation of the compliant walls, as velocity scale the maximum velocity of the steady plane Poiseuille flow, $U_{0}^{*}$, while the forcing angular frequency of the periodic pulsations defines the time scale, $1 / \omega_{f}^{*}$ (when the forcing frequency tends to zero a characteristic time scale can be defined through the velocity, $\left.U_{0}^{*}\right)$. The flow field is then characterized by the Reynolds number, $R e=U_{0}^{*} L^{*} / \nu_{l}^{*}$, where $\nu_{l}^{*}$ the kinematic viscosity 


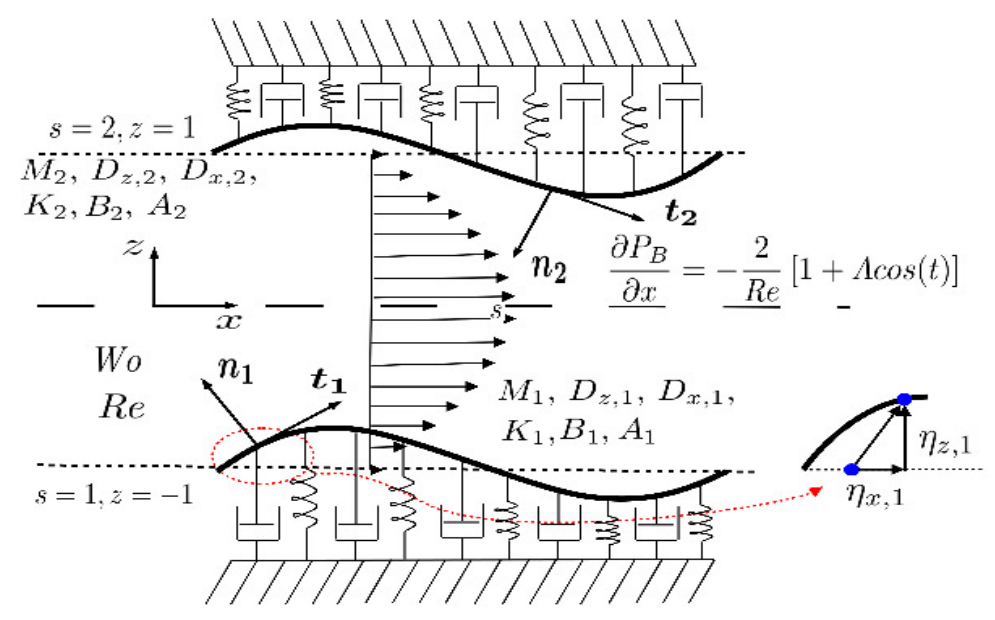

FiguRE 1. Schematic of the system studied

of the fluid, and the Womersley number, $W_{o}=L^{*}\left(\omega_{f}^{*} / \nu_{l}^{*}\right)^{1 / 2}$ with the Navier-Stokes equations taking the form,

$$
\frac{W o^{2}}{R e} \frac{\partial \boldsymbol{U}}{\partial t}+\boldsymbol{U} \cdot \boldsymbol{\nabla} \boldsymbol{U}=-\boldsymbol{\nabla} P+\frac{1}{R e} \nabla^{2} \boldsymbol{U} .
$$

Here and hereafter, ${ }^{*}$ denotes a dimensional quantity. The fluid is assumed to move only in the streamwise direction $x$ due to a mean pressure gradient applied in this direction that changes periodically with a frequency $\omega_{f}^{*}$ and amplitude $\Lambda$,

$$
\frac{\partial P_{B}}{\partial x}=\frac{\mathrm{d} \bar{P}}{\mathrm{~d} x}\left(1+\Lambda \frac{\exp (\mathrm{i} t)+\exp (-\mathrm{i} t)}{2}\right) .
$$

Alternatively, periodic velocity variation with amplitude $\Lambda_{v}$ could be imposed; these relate to the amplitude of the disturbance pressure gradient through $\Lambda_{v}=2 \Lambda / W o^{2}$. Our choice of $\Lambda$ as the control parameter ensures that finite pumping power is maintained as we vary $W o$.

We assume that the mean pressure gradient does not induce vertical displacements of the compliant walls and so in their equilibrium state they rest along $z=\mp 1$. This assumption extends to the unsteady base flow provided that the vertical wall displacements induced by the fluctuating part of the pressure gradient are small. This is the case for the stiff walls used in the present study but its effect in yielding a timevarying channel height would need to be incorporated in the calculation of the unsteady base flow when the walls are very flexible. Correspondingly, for the compliant walls that admit axial displacement, we assume that the fluid shear of the base flow is cancelled by the inclusion of pre-tension in the wall.

Decomposing the base velocity field into steady and time-dependent parts, $\boldsymbol{U}_{B}(\boldsymbol{x}, t)=$ $\overline{\boldsymbol{U}(\boldsymbol{x})}+\boldsymbol{U}^{\prime}(\boldsymbol{x}, t)$, and taking into account that each part independently satisfies the continuity equation, the fluid momentum equation (2.1), the no-slip boundary conditions on the undeformed compliant walls, we obtain for the steady part,

$$
\bar{U}_{x}=1-z^{2} \quad \text { and } \quad \frac{\mathrm{d} \bar{P}}{\mathrm{~d} x}=-\frac{2}{R e}
$$


while the time-dependent part of the base flow is given by

$$
\begin{aligned}
U_{x}{ }^{\prime}=q_{-1} \exp (-\mathrm{i} t)+q_{1} \exp (\mathrm{i} t)= & \frac{\mathrm{i} \Lambda}{W o^{2}}\left[1-\frac{\cosh \left(\mathrm{i}^{3 / 2} W_{o} z\right)}{\cosh \left(\mathrm{i}^{3 / 2} W o\right)}\right] \exp (-\mathrm{i} t) \\
& +\frac{\mathrm{i} \Lambda}{W o^{2}}\left[\frac{\cosh \left(\mathrm{i}^{1 / 2} W o z\right)}{\cosh \left(\mathrm{i}^{1 / 2} W o\right)}-1\right] \exp (\mathrm{i} t),
\end{aligned}
$$

where $z$ is the coordinate normal to the flow direction.

\subsection{Perturbation field}

\subsubsection{Flow field}

We proceed by decomposing the velocity field to a base flow field and a field of smallamplitude disturbances which have a spatial exponential form,

$$
\begin{array}{r}
\boldsymbol{U}(x, y, z, t)=U_{x, B}(z, t)+\epsilon \boldsymbol{u}(x, y, z, t)=U_{x, B}(z, t)+\epsilon \hat{\boldsymbol{u}}(z, t) \exp (\mathrm{i} \alpha x+\mathrm{i} \beta y)+\text { c.c. }, \\
P(x, y, z, t)=P_{B}(x, t)+\epsilon p(x, y, z, t)=P_{B}(x, t)+\epsilon \hat{p}(z, t) \exp (\mathrm{i} \alpha x+\mathrm{i} \beta y)+\text { c.c. },
\end{array}
$$

where $\epsilon \ll 1, \alpha, \beta$ are the disturbance wavenumbers in the streamwise and spanwise direction respectively, while the amplitudes of disturbance velocity and pressure, $\hat{\boldsymbol{u}}(z, t)$, $\hat{p}(z, t)$ depend on time and c.c. denotes the complex conjugate. Substituting the perturbation forms of equation (2.5) into the governing equation (2.1) and the continuity equation, and expanding in powers of $\epsilon$, we obtain for the $O(\epsilon)$ problem, after subtracting the $O(1)$ problem and eliminating the pressure disturbance,

$$
W o^{2} \frac{\partial}{\partial t} \mathcal{L} \hat{u}_{z}=\left(\mathcal{L}-\mathrm{i} \alpha \operatorname{Re} U_{x, B}\right) \mathcal{L} \hat{u}_{z}+\mathrm{i} \alpha \operatorname{Re} \frac{\partial^{2} U_{x, B}}{\partial z^{2}} \hat{u}_{z},
$$

where $\mathcal{L}=\partial^{2} / \partial z^{2}-\kappa^{2}$ and $\kappa^{2}=\alpha^{2}+\beta^{2}$. Equation (2.6) is recognised as the Orr-Sommerfeld equation for pulsatile flow that supports the well-known flow-based inflectional and Tollmien-Schlichting-wave instabilities and their inter-connectedness that feature in the results that follow.

Using decomposition (2.5), the continuity equation, the streamwise and spanwise part of the Navier-Stokes equations for the $O(\epsilon)$ problem, we can express the pressure disturbance amplitude $\hat{p}(z, t)$ as a function of $\hat{u}_{z}(z, t)$,

$$
\hat{p}=-\frac{W o^{2}}{\kappa^{2} \operatorname{Re}} \frac{\partial^{2} \hat{u}_{z}}{\partial t \partial z}-\frac{\mathrm{i} \alpha U_{x, B}}{\kappa^{2}} \frac{\partial \hat{u}_{z}}{\partial z}+\frac{\mathrm{i} \alpha}{\kappa^{2}} \frac{\partial U_{x, B}}{\partial z} \hat{u}_{z}+\frac{1}{\kappa^{2} R e} \mathcal{L} \frac{\partial \hat{u}_{z}}{\partial z} .
$$

\subsubsection{Structural model}

For the compliant-wall dynamics, we use the two-dimensional isotropic Kirchhoff plate equation with additional terms to account for a dashpot-type damping and a uniformly distributed spring foundation. Combined with the normal force balance on the two compliant walls (Shankar \& Kumaran 2002) and keeping only $O(\epsilon)$ terms, we obtain

$$
\begin{aligned}
\boldsymbol{n}_{s} \cdot \boldsymbol{T} \cdot \boldsymbol{n}_{s} & =-p(x, y, z=\mp 1, t)+\frac{2}{R e} \frac{\partial u_{z}}{\partial z}(x, y, z=\mp 1, t) \mp \frac{2}{R e} \frac{\partial U_{x, B}}{\partial z}(z=\mp 1, t) \frac{\partial \eta_{z, s}}{\partial x} \\
& =M_{s} \frac{\partial^{2} \eta_{z, s}}{\partial t^{2}}+D_{z, s} \frac{\partial \eta_{z, s}}{\partial t}+K_{s} \eta_{z, s}+B_{s}\left[\frac{\partial^{4} \eta_{z, s}}{\partial x^{4}}+2 \frac{\partial^{4} \eta_{z, s}}{\partial x^{2} y^{2}}+\frac{\partial^{4} \eta_{z, s}}{\partial y^{4}}\right], \quad(2.8)
\end{aligned}
$$

where $\boldsymbol{n}_{s}=-\left(\partial \eta_{z, s} / \partial x\right) \boldsymbol{e}_{\boldsymbol{x}}-\left(\partial \eta_{z, s} / \partial y\right) \boldsymbol{e}_{\boldsymbol{y}} \pm \boldsymbol{e}_{\boldsymbol{z}}$ are the linearised unit vectors normal 
to the two compliant surfaces ( $s=1,2$ lower/upper surfaces) and directed into the fluid domain, $\boldsymbol{T}=-P \boldsymbol{I}+(1 / R e)\left[\nabla \boldsymbol{U}+(\nabla \boldsymbol{U})^{T}\right]$ is the total stress tensor of the fluid at the two compliant walls, $\eta_{z, s}(x, y, t)$, the vertical displacement of the two compliant surfaces, considered positive in the direction of the normal vectors, and $p(x, y, z=$ $\mp 1, t)$ is the non-dimensional pressure disturbance on the compliant walls. The nondimensional coefficients of compliant-wall inertia, damping, spring-foundation stiffness, flexural rigidity respectively, are defined by

$$
M_{s}=\frac{\rho_{s}^{*} h_{s}^{*} \omega_{f}^{* 2} L^{*}}{\rho_{l}^{*} U_{0}^{* 2}}, \quad D_{z, s}=\frac{D_{z, s}^{*} \omega_{f}^{*} L^{*}}{\rho_{l}^{*} U_{0}^{* 2}}, \quad K_{s}=\frac{K_{s}^{*} L^{*}}{\rho_{l}^{*} U_{0}^{* 2}}, \quad B_{s}=\frac{B_{s}^{*}}{\rho_{l}^{*} U_{0}^{* 2} L^{* 3}},
$$

with $\rho_{s}^{*}$ and $h_{s}^{*}$ the material density and thickness, respectively and $B_{s}^{*}=E_{s}^{*} h_{s}^{* 3} /[12(1-$ $\left.\nu^{2}\right)$ ] with $E_{s}^{*}$ the elastic modulus and $\nu$ the Poisson ratio (Timoshenko \& WoinowskyKrieger 1959).

\subsubsection{Interface conditions}

On the compliant surfaces, the perturbation velocity and stresses are each continuous between fluid and solid. Thus, the boundary conditions for the velocity for the $O(\epsilon)$ problem are

$$
\begin{array}{r}
u_{x}(x, y, z=\mp 1, t) \pm \eta_{z, s}(x, y, t) \frac{\partial U_{x, B}}{\partial z}(z=\mp 1, t)=0, \\
u_{y}(x, y, z=\mp 1, t)=0,
\end{array}
$$

Using the decomposition $\eta_{z, s}(x, y, t)=\hat{\eta}_{z, s}(t) \exp (\mathrm{i} \alpha x+\mathrm{i} \beta y)+$ c.c. and $(2.5)$ in $(2.10)$ and (2.8), taking into account (2.7) and the continuity equation, we obtain the first boundary condition for the $O(\epsilon)$ problem applied at the compliant surfaces $(z=\mp 1)$,

$$
\begin{array}{r} 
\pm W o^{4} \alpha \frac{\partial U_{x, B}}{\partial z} \frac{\partial^{2} \hat{u}_{z}}{\partial t \partial z}-\kappa^{2} R e^{2} \alpha \frac{\partial U_{x, B}}{\partial z} M_{s} \frac{\partial \hat{u}_{z}}{\partial t}= \\
\kappa^{2} R e^{2} \alpha \frac{\partial U_{x, B}}{\partial z} D_{z, s} \hat{u}_{z}-\mathrm{i} \kappa^{2} W o^{2} R e\left[B_{s}\left(\alpha^{4}+2 \alpha^{2} \beta^{2}+\beta^{4}\right)+K_{s}\right] \frac{\partial \hat{u}_{z}}{\partial z} \\
\pm \mathrm{i} \alpha^{2} W o^{2} R e \frac{\partial U_{x, B}}{\partial z}\left(\frac{\partial U_{x, B}}{\partial z} \hat{u}_{z}-U_{x, B} \frac{\partial \hat{u}_{z}}{\partial z}\right) \\
\pm W o^{2} \alpha \frac{\partial U_{x, B}}{\partial z}\left(\frac{\partial^{3} \hat{u}_{z}}{\partial z^{3}}-\kappa^{2} \frac{\partial \hat{u}_{z}}{\partial z}\right)
\end{array}
$$

while the enforcement of (2.10)(c), taking into account (2.10)(a) and the continuity equation, yields the second boundary condition on the compliant surfaces $(z=\mp 1)$,

$$
\mathrm{i} W o^{2} \frac{\partial U_{x, B}}{\partial z} \frac{\partial^{2} \hat{u}_{z}}{\partial t \partial z}=\mathrm{i} W o^{2} \frac{\partial^{2} U_{x, B}}{\partial t \partial z} \frac{\partial \hat{u}_{z}}{\partial z}-\alpha R e\left(\frac{\partial U_{x, B}}{\partial z}\right)^{2} \hat{u}_{z}
$$

\subsubsection{Spatial discretization}

For spatial discretization in the direction normal to the base flow, $z$, we use the Chebyshev pseudospectral method. The domain is discretized into $M+1$ collocation points, with their positions given by

$$
z(j)=-\cos \left[\frac{(j-1) \pi}{M}\right], \text { for } \quad j=1, M+1,
$$


and the spatial derivatives of a function at the collocation points are then related to the values of the function there through the Chebyshev differentiation matrices (Canuto et al. 1988).

\section{FSI accounting for both vertical and axial structural displacements}

Herein, the FSI system for plate-spring compliant walls that admit both vertical and axial displacements is modelled for two-dimensional (streamwise and normal to the flow) disturbances. In addition to the normal force balance, equation (2.8), we enforce the tangential force balance on the compliant walls (Shankar \& Kumaran 2002); this provides the equation of motion for axial displacements,

$$
\begin{aligned}
\boldsymbol{t}_{s} \cdot \boldsymbol{T} \cdot \boldsymbol{n}_{s}= & \pm \frac{1}{R e}\left(\frac{\partial u_{z}}{\partial x}(x, z=\mp 1, t)+\frac{\partial u_{x}}{\partial z}(x, z=\mp 1, t)\right)= \\
& M_{s} \frac{\partial^{2} \eta_{x, s}}{\partial t^{2}}+D_{x, s} \frac{\partial \eta_{x, s}}{\partial t}-A_{s} \frac{\partial^{2} \eta_{x, s}}{\partial x^{2}},
\end{aligned}
$$

where, $\boldsymbol{t}_{s}=\boldsymbol{e}_{\boldsymbol{x}} \pm\left(\partial \eta_{z, s} / \partial x\right) \boldsymbol{e}_{\boldsymbol{z}}$, are the linearised unit vectors tangential to the compliant surface, $\eta_{x, s}(x, t)$, is the dimensionless axial wall displacement, $D_{x, s}$ is the dimensionless structural damping in the streamwise direction, and $A_{s}$ the dimensionless in-plane stiffness (the resistance of the compliant walls to the change of the axial deformations) defined as $A_{s}=E_{s}^{*} h_{s}^{*} /\left[\left(1-\nu^{2}\right)\left(\rho_{l}^{*} U_{0}^{* 2} L^{*}\right)\right]$.

The linearised kinematic boundary conditions are written as

$$
\begin{aligned}
u_{x}(x, z=\mp 1, t) \pm \eta_{z, s}(x, t) \frac{\partial U_{x, B}}{\partial z}(z=\mp 1, t) & =\frac{W o^{2}}{R e} \frac{\partial \eta_{x, s}}{\partial t}(x, t), \quad(a) \\
u_{z}(x, z=\mp 1, t) & = \pm \frac{W o^{2}}{R e} \frac{\partial \eta_{z, s}}{\partial t}(x, t)
\end{aligned}
$$

Using the decompositions (2.5) with $\beta=0, \eta_{z, s}(x, t)=\hat{\eta}_{z, s}(t) \exp (\mathrm{i} \alpha x)+$ c.c. and $\eta_{x, s}(x, t)=\hat{\eta}_{x, s}(t) \exp (\mathrm{i} \alpha x)+$ c.c., equation (2.7) and the continuity equation, the system of equations (2.6) with the boundary conditions (2.8), (3.1), (3.2) is cast in the form of the following initial value problem,

$$
\frac{d \hat{\boldsymbol{v}}}{d t}=\boldsymbol{P}(t) \hat{\boldsymbol{v}}, \quad \hat{\boldsymbol{v}}=\left\{\hat{c}_{z, s}, \hat{\eta}_{z, s}, \hat{c}_{x, s}, \hat{\eta}_{x, s}, \hat{\boldsymbol{u}}_{z}^{T}\right\}^{T}, \quad \hat{\boldsymbol{v}}(t=0)=\hat{\boldsymbol{v}}^{0}
$$

where $\hat{c}_{z, s}=\mathrm{d} \hat{\eta}_{z, s} / \mathrm{d} t, \hat{c}_{x, s}=\mathrm{d} \hat{\eta}_{x, s} / \mathrm{d} t$ and $\boldsymbol{P}$ is periodic in time with fundamental period $T$.

\section{Methods of solution}

4.1. Floquet analysis through decomposition into a product of exponential growth and sum of harmonics

Following Thomas et al. (2011), the unknown variable $\hat{u}_{z}(z, t)$ is decomposed into a product of exponential growth and a sum of harmonics,

$$
\hat{u}_{z}(z, t)=\exp (\mu t) \sum_{n=-N}^{N} \phi_{n}(z) \exp (\mathrm{i} n t),
$$

where $\mu$ are the Floquet exponents whose real values define temporal instability $(+$ ve) 
/ stability(-ve) of the system and $N$ is a large number, typically $N=1000$, in order to ensure a converged solution. Substituting (4.1) into (2.6) and equating coefficients of harmonics results in

$$
\begin{aligned}
\mu W o^{2} \mathcal{L} \phi_{n}= & -\mathrm{i} \alpha \operatorname{Re}\left(q_{1} \mathcal{L}-\frac{\partial^{2} q_{1}}{\partial z^{2}}\right) \phi_{n-1} \\
& +\left(\mathcal{L}^{2}-\mathrm{i} W o^{2} n \mathcal{L}-\mathrm{i} \alpha \operatorname{Re} \bar{U}_{x, B} \mathcal{L}+\mathrm{i} \alpha \operatorname{Re} \frac{\partial^{2} \bar{U}_{x, B}}{\partial z^{2}}\right) \phi_{n} \\
& -\mathrm{i} \alpha \operatorname{Re}\left(q_{-1} \mathcal{L}-\frac{\partial^{2} q_{-1}}{\partial z^{2}}\right) \phi_{n+1} .
\end{aligned}
$$

Relevant expressions for the boundary conditions are obtained by substituting (4.1) into (2.11) and (2.12). The system of equations comprising (4.2) together with the boundary conditions, is cast as the generalized eigenvalue problem,

$$
\begin{aligned}
& \boldsymbol{A} \boldsymbol{y}=\mu \boldsymbol{B} \boldsymbol{y}, \\
& \boldsymbol{y}=\left\{\begin{array}{lllllll}
\boldsymbol{\phi}_{-N}^{T} & \boldsymbol{\phi}_{-N+1}^{T} & \ldots & \boldsymbol{\phi}_{0}^{T} & \ldots & \boldsymbol{\phi}_{N-1}^{T} & \boldsymbol{\phi}_{N}^{T}
\end{array}\right\}^{T},
\end{aligned}
$$

where $\boldsymbol{A}$ and $\boldsymbol{B}$ are sparse matrices and $\boldsymbol{\phi}$ the vector of $M+1$ unknowns arising from the spatial discretization. Typically, $M=70$ in order to obtain convergence. The function eigs in MATLAB was implemented to extract the eigenvalues $\mu$ and eigenvectors $\boldsymbol{y}$.

\subsection{Floquet analysis through the monodromy matrix}

The system of equations (2.6), with the boundary conditions (2.11) and (2.12) can be written in a similar form to the initial value problem (3.3),

$$
\frac{d \hat{\boldsymbol{u}}_{z}}{d t}=\boldsymbol{Q}(t) \hat{\boldsymbol{u}}_{z}, \quad \hat{\boldsymbol{u}}_{z}(t=0)=\hat{\boldsymbol{u}}_{z}^{0},
$$

where the matrix $\boldsymbol{Q}$ is periodic with known period $T$, due to the imposed periodic base velocity $\boldsymbol{U}_{B}$. The monodromy matrix $\boldsymbol{M}$ is calculated by solving the following initial value matrix problem (Iooss \& Joseph 1990; Seydel 1988):

$$
\boldsymbol{M}=\boldsymbol{S}(t=T): \quad \frac{d \boldsymbol{S}}{d t}=\boldsymbol{Q}(t) \boldsymbol{S}, \quad \boldsymbol{S}(t=0)=\boldsymbol{I},
$$

where $\boldsymbol{I}$ is the identity matrix, $\boldsymbol{S}(t)=\boldsymbol{W}(t) \exp (t \boldsymbol{C})$, the fundamental matrix, $\boldsymbol{W}(t+$ $T)=\boldsymbol{W}(t), T$-periodic matrix, while the eigenvalues of the monodromy matrix $\boldsymbol{M}=$ $\exp (T \boldsymbol{C})=\boldsymbol{Z} \exp (T \boldsymbol{D}) \boldsymbol{Z}^{-1}$, called Floquet multipliers $k$, define the system stability and $\boldsymbol{D}$ the diagonal matrix with components being the Floquet exponents $\mu$ (Schmid 2007); hence $k=\exp (T \mu)$. Finally, the columns of $\boldsymbol{Z}$ contain the eigenvectors of the matrix $\boldsymbol{C}$, that correspond to the Floquet exponents. Using a second-order backward-integration scheme for time evolution we obtain at time $n$,

$$
\begin{array}{r}
\boldsymbol{S}^{n}=\left[3 \boldsymbol{I}-2 \Delta t \boldsymbol{Q}^{n}\right]^{-1}\left[4 \boldsymbol{S}^{n-1}-\boldsymbol{S}^{n-2}\right], \\
\boldsymbol{S}^{0}=\boldsymbol{S}(t=0)=\boldsymbol{I}, \quad \boldsymbol{S}^{1}=\left[\boldsymbol{I}-\Delta t \boldsymbol{Q}^{1}\right]^{-1} \boldsymbol{S}^{0}, \quad \boldsymbol{M}=\boldsymbol{S}(t=T)=\boldsymbol{S}^{N},
\end{array}
$$

where we have used for $n=1$, the first order backward Euler scheme to initiate the iteration procedure. Alternatively, (4.5) can be integrated explicitly in time using the 
following iterative algorithm for the solution of the fundamental matrix $\mathbf{S}$ (Schmid \& Kytomaa 1994)

$$
\boldsymbol{S}_{l+1}^{n}=\boldsymbol{I}+\int_{0}^{n} \boldsymbol{Q}^{n} \boldsymbol{S}_{l}^{n} \mathrm{~d} t,
$$

where the integral of the matrices, calculated with the trapezoidal rule, is taken component-wise and the solution at time $n$ is considered converged after the $(l+1)$ th iteration, when the Frobenius norm of the matrix corresponding to the relative error between consecutive iterations, becomes smaller than a specified value. Following either of the above two numerical procedures for the solution of (4.5), the matrix $\boldsymbol{M}$ is evaluated at the end of one period $T$ from the product of the matrices or the sum of the integrals calculated at the previous times, respectively. Its eigenvalues, the Floquet multipliers, $k_{j}, j=1, \cdots M+1$ determine the stability of the pulsatile flow through the compliant channel in the following manner: The flow-structure system is stable if $\left|k_{j}\right|<1$ for $j=1,2, \cdots M+1$ and unstable if $\left|k_{j}\right|>1$ for some $j$ (Iooss \& Joseph 1990; Tsiglifis \& Pelekasis 2011). In addition, the sign of the real part of the unstable eigenvalue, determines how the resulting solution is unstable: if $\operatorname{Im}\left(k_{j}\right)=0$ with $\operatorname{Re}\left(k_{j}\right)<-1$ then the unstable solution, $\hat{\boldsymbol{u}}_{z}(t)$, is periodic with twice the period of that of the base flow, whereas if $\operatorname{Im}\left(k_{j}\right)=0$ with $\operatorname{Re}\left(k_{j}\right)>1$ the solution is periodic with the same period of the base flow. Finally, if $\operatorname{Im}\left(k_{j}\right) \neq 0$ with $\left|k_{j}\right|>1$ the unstable solution is quasi-periodic with characteristic frequencies being those of the base flow and the bifurcating solution, leading to a toroidal structure in the phase space (Iooss \& Joseph 1990).

\subsection{Modal transient growth}

The preceding analysis determines whether disturbances amplify in the limit of an infinite number of periods of oscillation. However, within the period, $T$, of a single pulsation, that features inflectional velocity profiles, large modal transient growth of disturbance kinetic energy may occur. This can be advected downstream during, potentially causing transition to turbulence even though all the Floquet multipliers lie within the unit disk. We are therefore motivated to assess the efficacy of compliant walls in mitigating the total transient energy growth as compared to that generated in a rigid channel. The solution of the systems (4.4) or (3.3) can be written as,

$$
\hat{\boldsymbol{u}}(t)=\boldsymbol{S}(t) \hat{\boldsymbol{u}}_{z}^{0}
$$

Then, the transient growth of the above system at time $t$ maximized over all the initial conditions, quantified by the induced 2 -norm of the fundamental matrix $\boldsymbol{S}$, is:

$$
\begin{aligned}
G(t) & =\max _{\hat{\boldsymbol{u}}_{z}^{0} \neq 0} \frac{\|\hat{\boldsymbol{u}}(t)\|_{2}^{2}}{\left\|\hat{\boldsymbol{u}}_{z}^{0}\right\|_{2}^{2}}=\max _{\hat{\boldsymbol{u}}_{z}^{0} \neq 0} \frac{\left\|\boldsymbol{S}(t) \hat{\boldsymbol{u}}_{z}^{0}\right\|_{2}^{2}}{\left\|\hat{\boldsymbol{u}}_{z}^{0}\right\|_{2}^{2}}=\|\boldsymbol{S}(t)\|_{2}^{2}=\sigma_{\max }^{2}(\boldsymbol{S}(t)) \\
& =\max _{\hat{\boldsymbol{u}}_{z}^{0} \neq 0} \frac{\hat{\boldsymbol{u}}_{z}^{\dagger}(t) \hat{\boldsymbol{u}}_{z}(t)}{\hat{\boldsymbol{u}}_{z}^{\dagger 0} \hat{\boldsymbol{u}}_{z}^{0}}=\max _{\hat{\boldsymbol{u}}_{z}^{0} \neq 0} \frac{\hat{\boldsymbol{u}}_{z}^{\dagger 0} \boldsymbol{S}^{\dagger}(t) \boldsymbol{S}(t) \hat{\boldsymbol{u}}_{z}^{0}}{\hat{\boldsymbol{u}}_{z}^{\dagger 0} \hat{\boldsymbol{u}}_{z}^{0}},
\end{aligned}
$$

where $\sigma_{\max }$ is the largest singular value, $\dagger$ is the conjugate transpose and the quotient is recognized as the Rayleigh quotient (Meyer 2000; Schmid \& Henningson 2001). The maximum total growth, $G(t)$, at time t, is obtained by finding the largest singular value of the fundamental matrix through SVD (Singular Value Decomposition) or by solving the following eigenproblem for the largest eigenvalue $\lambda_{\max }$ (Mao et al. 2009; Meyer 2000; Schmid \& Henningson 2001), 


$$
\mathbf{S}^{\dagger}(t) \boldsymbol{S}(t) \hat{\boldsymbol{u}}_{z}^{0}=\lambda \hat{\boldsymbol{u}}_{z}^{0}
$$

An alternative way to measure the transient growth is by integrating numerically the initial-value problems, (4.4) or (3.3), using a random initial vector $\hat{\boldsymbol{v}}^{0}$ (Kerczek 1982) and calculating the time evolution of the total energy (TE) of the fluid-structure interaction (FSI), written here for the two-dimensional case and when both vertical and axial displacements of the compliant walls occur as

$$
\begin{aligned}
E(t)= & \frac{1}{2} \int_{-\pi / \alpha}^{\pi / \alpha} \int_{-1}^{1}\left|u_{x}\right|^{2}+\left|u_{z}\right|^{2} \mathrm{~d} z \mathrm{~d} x \\
& +\frac{1}{2} \int_{-\pi / \alpha}^{\pi / \alpha} M_{s}\left(\frac{\partial \eta_{z, s}}{\partial t}\right)^{2}+M_{s}\left(\frac{\partial \eta_{x, s}}{\partial t}\right)^{2} \mathrm{~d} x \\
& +\frac{1}{2} \int_{-\pi / \alpha}^{\pi / \alpha} B_{s}\left(\frac{\partial^{2} \eta_{z, s}}{\partial x^{2}}\right)^{2}+A_{s}\left(\frac{\partial \eta_{x, s}}{\partial x}\right)^{2}+K_{s} \eta_{z, s}^{2} \mathrm{~d} x .
\end{aligned}
$$

The first and second integrals respectively correspond to the flow (FKE) and structural kinetic energies (SKE), while the third evaluates the strain energy of the structure (SPE) comprising contributions from plate flexure, plate tension and the spring foundation, respectively in the order written.

Finally, we remark that details of the calculation of non-modal transient growth are deferred to $\S 5.3$ in which this mechanism is explored in the context of time-asymptotic stability and modal transient growth.

\section{Results and Discussion}

\subsection{Steady plane Poiseuille flow}

In order to validate the present models, we consider steady Poiseuille flow between two parallel compliant walls with system parameters identical to those given by Eqn. (4.1) in the two-dimensional analysis of Davies \& Carpenter (1997b) wherein the critical Reynolds number and wavenumber were found, using local spatial stability analysis, to be $R e_{\text {crit. }}=6020.5$ and $\alpha_{\text {crit }} .=0.975$ for $\omega_{c}=\left(W o^{2} / R e\right) \omega=0.257$ (see also Figure (5) of Davies \& Carpenter (1997b)). We therefore use this Reynolds number and streamwise wavenumber, setting $\beta=0$ and $\Lambda=0$ in our model with $B_{s}=0.530, K_{s}=2.119$, $A_{s}=146.9$ and $M_{s}=5.75 \times 10^{-6}\left(M_{s}^{\prime}=0.333\right.$ when made dimensionless using the characteristic time-scale as defined through the centerline velocity) to generate the results presented in Fig. 2. We remark that for these and subsequent results of the eigen-analysis the variation of velocity perturbations, $u_{z}$ and $u_{x}$ and the shear stress, $\tau_{z x}$ across the channel height are normalised to unity; for clarity the eigenvectors of $u_{x}$ and $\tau_{z x}$ are plotted over the half-channel height because their variation is anti-symmetric about the channel centre-line.

Figure 2(a) shows the spectrum of Floquet exponents, with Fig. 2(b) being a magnification of the region around the least stable eigenvalues, predicted by the decomposition method for compliant walls with only vertical structural displacements. The onset of instability, evidenced by the marginally positive $\operatorname{Re}(\mu)$, demonstrates that the critical Reynolds number is closely aligned with that found by local spatial stability analysis. It is noted that the Floquet exponents are not unique in the sense that the $\mu=$ 
$\operatorname{Re}(\mu)+\mathrm{i}[\operatorname{Im}(\mu)+m]$ for integer $m$ are also eigenvalues; for this reason, the horizontal distance between the eigenvalues is unity.

Figure 2(c), is a plot of the time dependence of the normal component of the flow disturbance velocity, $u_{z}$, in the center of the channel (dashed blue line) and on the lower compliant wall (solid red line). It can be seen that the disturbance velocity oscillates but its amplitude remains constant with time thereby denoting neutral stability at the chosen (critical) Reynolds number. The oscillation frequency is determined by the spectral density of the time dependence of $u_{z}$ at the channel centre presented in Fig. $2(\mathrm{~d})$; the value of $\omega=62$ yields $\omega_{c}=0.257$ which agrees with that of Davies \& Carpenter $(1997 b)$ for steady Poiseuille flow. Figure 2(e) shows the eigenvector of disturbance shear stresses $\tau_{z x}=(1 / R e)\left(\partial u_{z} / \partial x+\partial u_{x} / \partial z\right)$ for the compliant- and rigid-wall cases. Careful comparison, reveals that wall compliance serves to increase marginally the concentration of fluid shear stress close to the wall-flow interface that would then suggest that the compliant wall experiences a slightly higher shear stress than its rigid counterpart.

Finally, Fig. 2(f) is a plot of the length of the Floquet multipliers against their imaginary part, extracted by the monodromy matrix, considering compliant-wall models with each of vertical and vertical-axial displacements. It can be seen that one system eigenvalue (highlighted by the arrow) has marginally crossed the unit disk thereby validating the results predicted by the decomposition method. The figure also shows that the inclusion of axial motion in the compliant-wall dynamics has almost no effect on the stability of the fluid-structure interaction for steady Poiseuille flow. It is remarked that the model which allows both vertical and axial deformations of the compliant walls has, through the construction of the Monodromy matrix, four real Floquet multipliers with length unity which appear as single point in the figure. These eigenvalues appear as a consequence of analytically taking the time derivative of the two equations (3.2)(a) and (b) at each boundary in order to couch the system in the form (4.5). Alternatively, we can retain the original system in the form

$$
\boldsymbol{L}(t) \frac{d \boldsymbol{S}}{d t}=\boldsymbol{R}(t) \boldsymbol{S}, \quad \boldsymbol{S}(t=0)=\boldsymbol{I}
$$

and after applying the second-order backward-integration scheme, we obtain the discretised solution equivalent to that of equation (4.6),

$$
\begin{array}{r}
\boldsymbol{S}^{n}=\left[3 \boldsymbol{L}^{n}-2 \Delta t \boldsymbol{R}^{n}\right]^{-1} \boldsymbol{L}^{n}\left[4 \boldsymbol{S}^{n-1}-\boldsymbol{S}^{n-2}\right], \\
\boldsymbol{S}^{0}=\boldsymbol{S}(t=0)=\boldsymbol{I}, \quad \boldsymbol{S}^{1}=\left[\boldsymbol{L}^{1}-\Delta t \boldsymbol{R}^{1}\right]^{-1} \boldsymbol{L}^{1} \boldsymbol{S}^{0} .
\end{array}
$$

\subsection{Pulsatile plane Poiseuille flow}

\subsubsection{Modulation frequency $W_{0}=5$}

We now focus on the stability of pulsatile Poiseuille flow between compliant walls when $W_{O}=5$. This frequency lies within the range of the frequencies encountered in the small vessels of human blood flow. Figures $3(\mathrm{a})$ and (b) show the velocity profiles of the pulsatile base flow for $\Lambda=3\left(\Lambda_{v}=2 \Lambda / W_{o}^{2}=0.24\right)$ and $\Lambda=5\left(\Lambda_{v}=0.4\right)$, respectively, during one period of the forcing disturbance pressure gradient. It can be seen that the flow is accelerated during the first half period of the forcing and is decelerated during the second, moving the inflection points away from the wall and towards the center of the channel.

Figure 4, is a plot of the variation of the Reynolds number at which the flow becomes unstable with the streamwise wavenumber for rigid and compliant walls and for different 
(a)

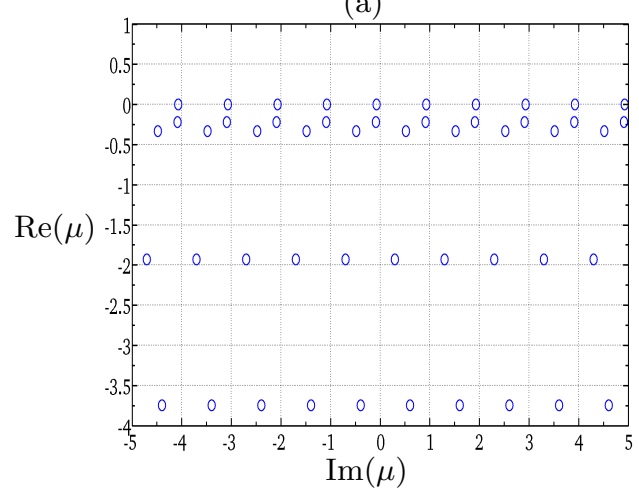

(c)

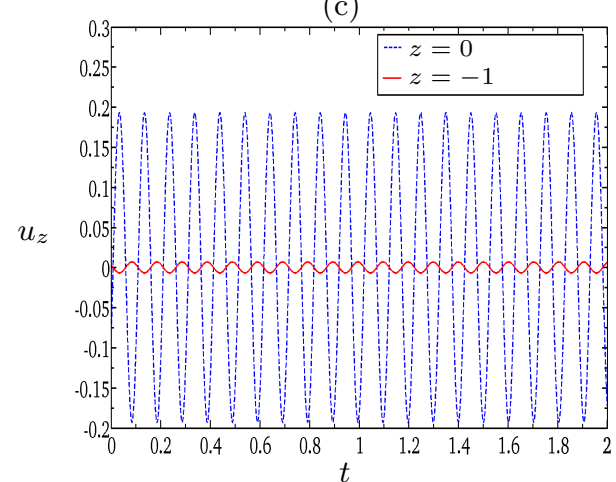

(e)

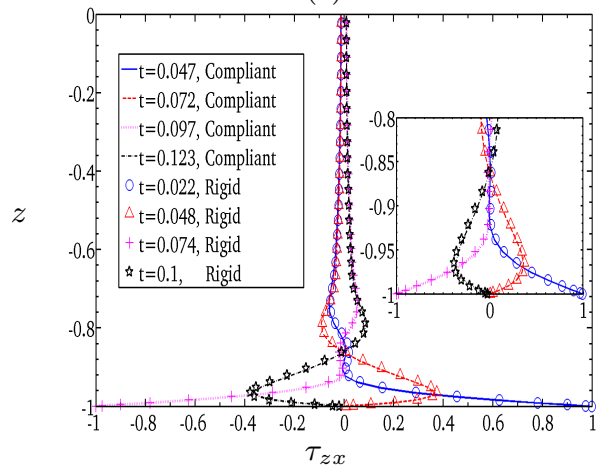

(b)

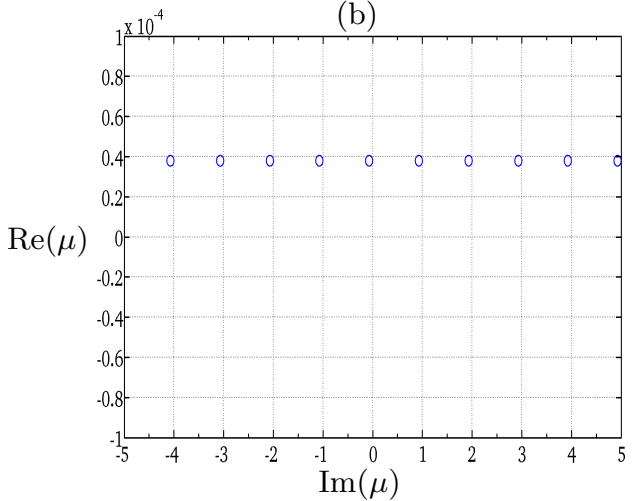

(d)

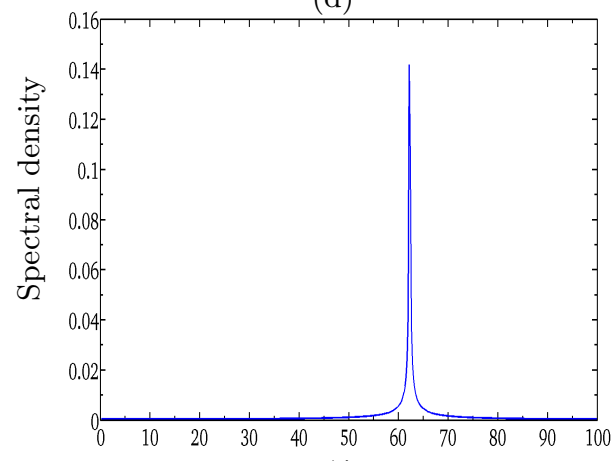

$(\mathrm{f})$

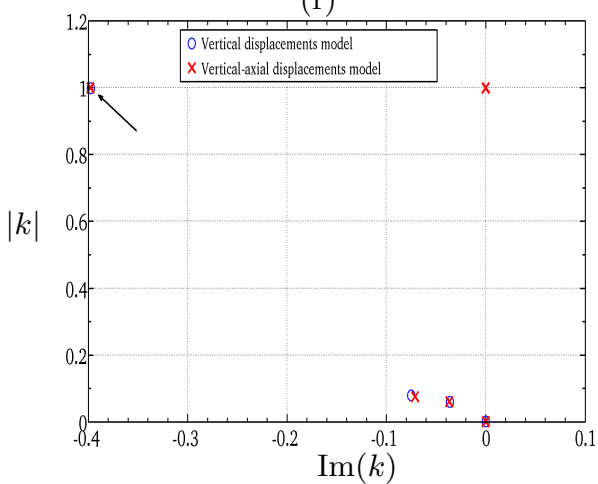

Figure 2. Steady plane Poiseuille flow through a compliant channel at instability onset: (a) Spectrum of the Floquet exponents for the vertical-displacement compliant-wall model, (b) Magnification of (a) to highlight the onset of instability, (c) Time-evolution of $u_{z}$ at the channel center-line (blue line) and at the lower compliant wall (red line), (d) Spectral density of the time sequence of $u_{z}$ at the channel center-line, (e) Perturbation shear stress $\tau_{z x}$ distribution across the channel over one period of oscillations, (f) Spectrum of the Floquet multipliers for the compliant-wall cases. Parameters: $R e_{\text {crit. }}=6020.5, \alpha_{\text {crit. }}=0.975, \beta=0, W_{o}=5, \Lambda=0$ with compliant-wall properties the same as those in Davies \& Carpenter (1997b)

values of the amplitude $\Lambda$ as predicted by the asymptotic analysis. It is seen that as the amplitude of the pulsation increases the flow is destabilized significantly relative to the underlying steady Poiseuille flow. This is in agreement with the theoretical work of Thomas et al. (2011), Singer et al. (1989), Kerczek (1982), Straatman et al. (2002) and the experimental observations of Nerem et al. (1972) and Einav \& Sokolov (1993), 
(a)

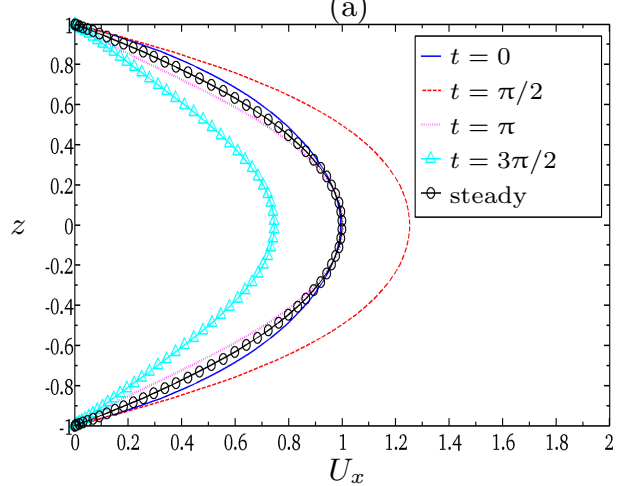

(b)

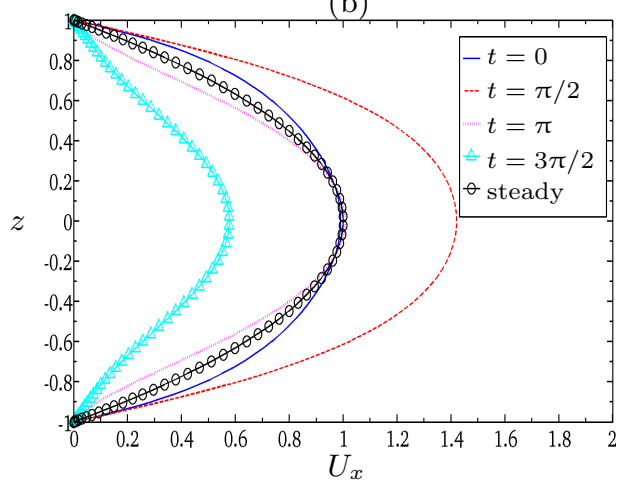

Figure 3. Pulsatile velocity profiles during one cycle of the periodic forcing pressure gradient with $W o=5$ for (a) $\Lambda=3\left(\Lambda_{v}=0.24\right)$ and (b) $\Lambda=5\left(\Lambda_{v}=0.4\right)$



FiguRe 4. Variation of Reynolds number at which the flow becomes unstable with streamwise wavenumber $\alpha$, for rigid and compliant walls and for different values of the modulation amplitude A. Parameters: $W o=5, \beta=0$, compliant wall properties same as defined in eq.(4.1) of Davies \& Carpenter $(1997 b)$

which showed that for low frequency modulation the flow is always destabilized due to the powerful inviscid (Rayleigh or inflectional) instability. It is also seen that the effect of wall compliance is stabilising in that it increases the critical Reynolds number (the minimum for which instability onset occurs) but that this effect diminishes as the amplitude of the flow pulsations is increased.

Figure 5(a), demonstrates convergence of the most unstable Floquet exponents of the flow over the compliant walls for $\Lambda=3, R_{\text {crit. }}=3775, \alpha_{\text {crit }}=1.1, \beta=0$ (the critical case indicated in figure 4 by a red arrow) with increasing number of collocation points as well as the number of Fourier modes, for the compliant-wall model accounting only for vertical displacements. It can be seen that a converged solution has been obtained for $\mathrm{N}=1000$ and $\mathrm{M}=71$. Figure 5(b), shows the corresponding Floquet multipliers for both the rigid and compliant-wall cases for models with and without the axial structural deformations. It can be deduced that the flow within rigid walls is 
slightly more asymptotically unstable than the flow within compliant walls and that the inclusion in the model of the axial structural deformations has a small stabilizing effect on the stability of the pulsatile flow through a compliant channel with its walls having the present properties. In addition, since $\operatorname{Im}\left(k_{j}\right) \neq 0$ with $\left|k_{j}\right|>1$ the unstable modes will have the frequencies both of the base flow and of the bifurcating solution. This can also be observed in figure 5(c) where we show the time-evolution of the disturbance velocity $u_{z}$ at the channel center-line and at the lower wall for the compliant-wall case. Figure $5(\mathrm{~d})$ is the spectral density of the channel centre-line $u_{z}$ disturbance for $\Lambda=0$ and $\Lambda=3$, for both the compliant- and rigid-wall cases. It can be seen that the unstable wave-packet contains the fundamental frequency of the base flow but the wave-packet comprises waves with broadband frequencies around the monochromatic frequency of the steady Poiseuille flow between rigid plates at the given Reynolds number.

We remark that for the compliant-wall properties used herein, the lowest in-vacuo eigenfrequency for vertical vibrations, $\omega_{v a c}=709.6$, is far too high to create resonance with the forcing frequency. The effect of wall compliance therefore has a very small effect on the distributions of axial-flow, $u_{x}$, and shear-stress, $\tau_{z x}$, perturbations across the channel as can be seen in figures $5(\mathrm{e})$ and (f). However, careful scrutiny shows that wall compliance increases the prominence of the single and double maxima that appear through the cycle of the eigenvector of $u_{x}$ and this effect generates a slightly increased concentration of $\tau_{z x}$ close to the wall as compared with the rigid-wall case.

Kerczek (1982) showed that disturbances which are predicted to be asymptotically stable at low Womersley number, could generate high levels of transient disturbance kinetic energy downstream during the fundamental period $T$ of pulsation due to the inflectional velocity profiles during the deceleration phase of the flow and this could trigger transition to turbulent flow. In figure 6(a) we show the modal transient growth of disturbances, $G$, during one cycle of oscillation for flow through rigid- and compliantwalled channels, the latter using the vertical-axial wall-displacement model. This figure also includes the ratio of the total energy of the FSI system, equation (4.11), relative to its initial value, $E(t) / E(t=0)$, as predicted using the integration of (3.3) for a random initial vector $\hat{\boldsymbol{v}}^{0}$ for both the compliant- and rigid-wall cases. We report that these results correspond to the third period of oscillation in order to discard artificial transient responses from the numerical integration of (3.3) (Kerczek 1982). It can be seen that both methods predict similar behaviour, most significantly a five-order increase to the magnitude of the disturbance total energy relative to its initial value and that the effect of wall-compliance is mildly mitigating. This large increase of the disturbance total energy during a part of the oscillation cycle for low modulation frequencies is in qualitative agreement with Kerczek (1982) and Singer et al. (1989) and occurs close to the time $t=3 \pi / 2$, when there is the maximum flow deceleration and the inflection points of the velocity profile are far from the walls.

In order to reveal how the total energy (TE) during modal transient growth is distributed during the oscillation cycle and demonstrate the effect of structural damping on it, we plot in figure 6(b) the time evolution of the system total energy (TE) and its components, namely, the flow kinetic energy (FKE), the structural kinetic energy (SKE) and the structural strain energy (SPE) for zero and at large values (that of critical damping $D_{\text {crit. }}=2 \sqrt{M_{s} K_{s}}$ ) of the structural damping as well as the case of flow in a rigid-walled channel. It can be seen that most of the total energy (TE) of the system comprises kinetic energy of the flow with a small contribution from the strain energy of the wall and negligible contribution from the structural kinetic energy. This reflects the fact that for the dynamics of the compliant wall given by its properties alongside the chosen modulation frequency, the wall-compliance exercises negligible stabilizing effect 
(a)

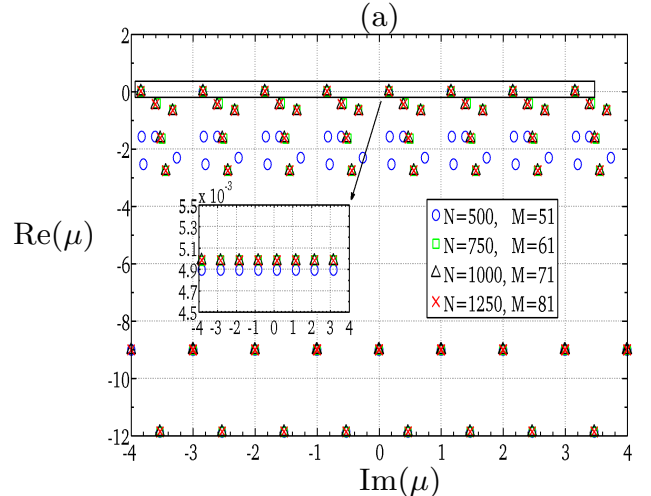

(c)

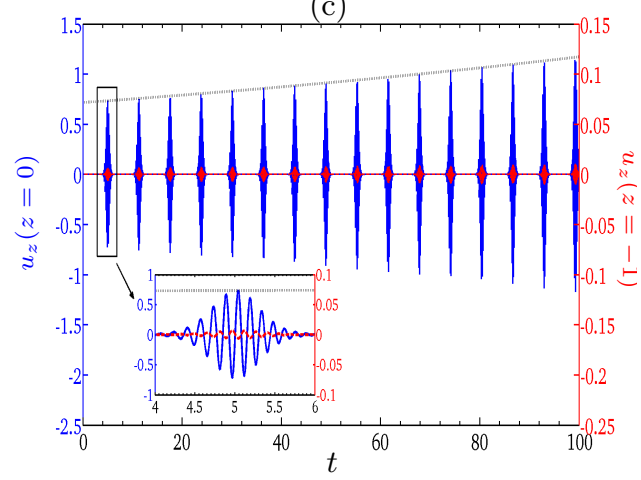

(e)

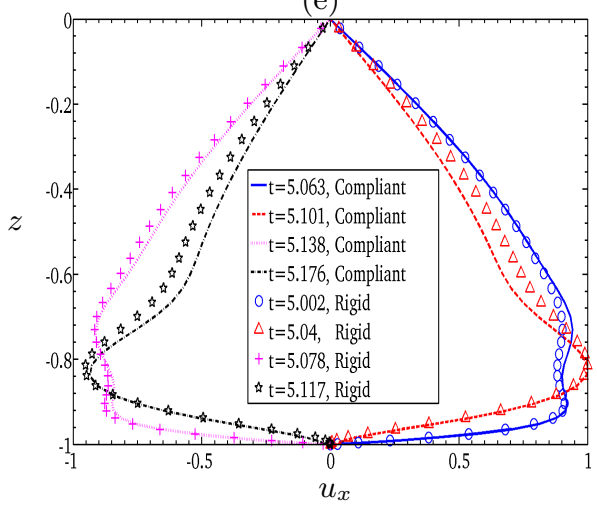

(b)

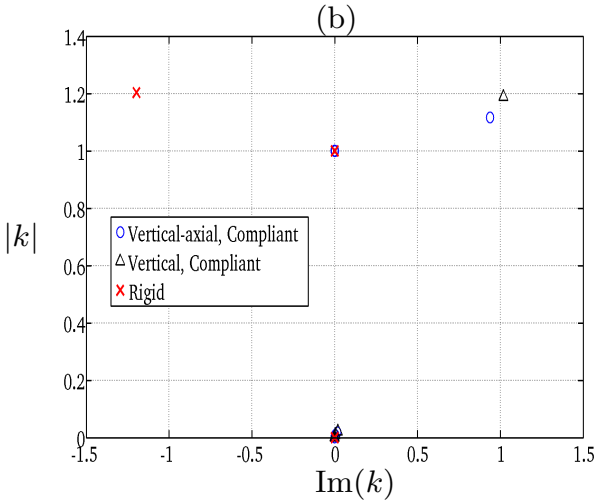

(d)

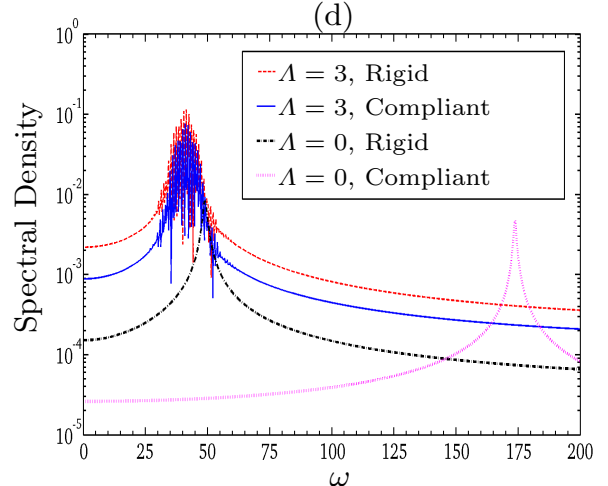

(f)

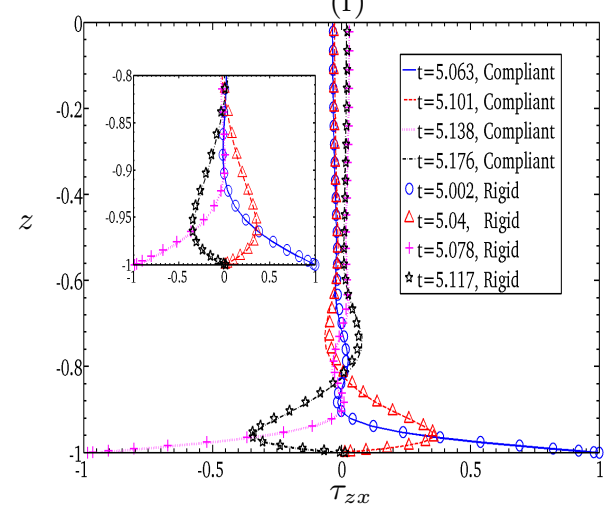

Figure 5. (a) Convergence of the Floquet exponents with increasing collocation points $(N)$ and Fourier modes $(M)$ for the compliant-wall case with vertical displacements, (b) Spectrum of the Floquet multipliers, (c) Time evolution of $u_{z}$ at the channel center-line (blue line) and at the bottom compliant wall (red line); the black dotted-line signifies the asymptotic solution, $\exp (\operatorname{Re}(\mu) t)$. (d) Spectral density of the time sequence of $u_{z}$ at the channel center-line, (e) $u_{x}$ and (f) $\tau_{z x}$ profiles during one cycle of oscillation. Parameters: Re $e_{\text {crit. }}=3775, \alpha_{\text {crit. }}=1.1$, $\beta=0, W_{o}=5, \Lambda=3$, compliant-wall properties same as defined in eq.(4.1) of Davies \& Carpenter $(1997 b)$

as also seen in the results of the asymptotic analysis in figure 4 for $\Lambda=3$. In addition, the inclusion of a large level of structural damping $D=D_{x, s}=D_{z, s}, s=1,2$ has a small destabilizing effect on the transient response which manifests itself by an almost one order of magnitude larger maximum flow kinetic energy than that found for the flow through a compliant channel without damping. 
(a)

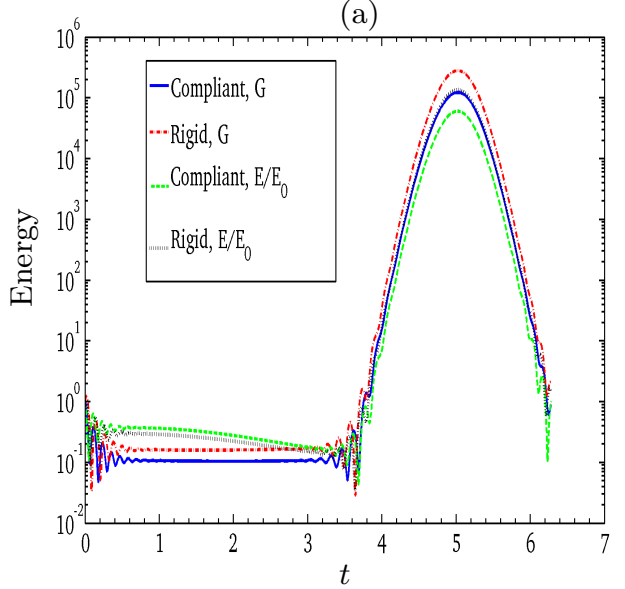

(b)

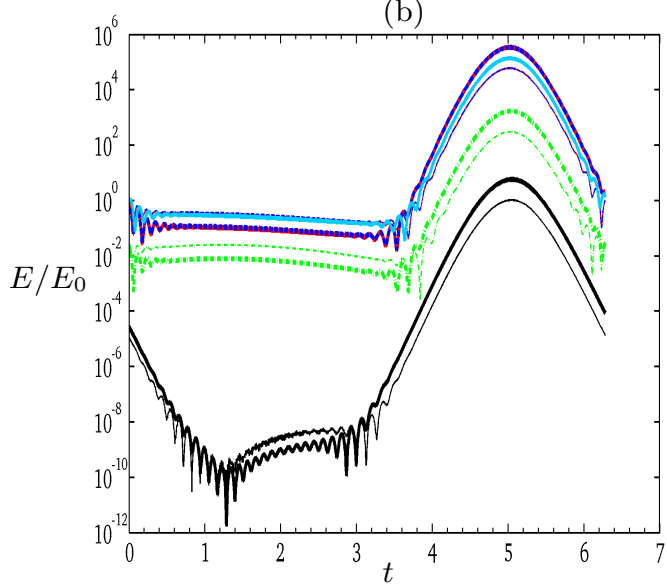

Legend for (b) (-Compliant, FKE, $\mathrm{D}=0),($-Compliant, $\quad \mathrm{SKE}, \quad \mathrm{D}=0)$, (- Compliant, SPE, $\mathrm{D}=0), \quad(-$-Compliant, TE, $\mathrm{D}=0), \quad(-$ Compliant, FKE, $\mathrm{D}=0.018),(-$ Compliant, $\quad \mathrm{SKE}, \quad \mathrm{D}=0.018),(-$ Compliant, $\quad \mathrm{SPE}, \quad \mathrm{D}=0.018)$, ( -Compliant, TE, D=0.018), ( Rigid, TE)

FIGURE 6. (a) Growth of disturbances during one cycle of modulation, $G$, and total energy ratio $E(t) / E(t=0)$ with time for compliant and rigid walls, (b) Time-evolution of the total energy (TE) and its contributions from the flow kinetic energy (FKE), structural kinetic energy (SKE) and structural strain energy (SPE) with and without structural damping $D=D_{x, s}=D_{z, s}, s=1,2$. Parameters: Re crit. $=3775, \alpha_{\text {crit. }}=1.1, \beta=0$, Wo $=5, \Lambda=3$, compliant-wall properties same as defined in eq. (4.1) of Davies \& Carpenter (1997b)

Cooper \& Carpenter (1997) studied the stability of rotating-disc boundary-layer flow over a compliant wall and found that structural damping has a destabilizing effect on the inviscid (Type I) inflectional instability and therefore this is categorised as a Class A instability in the energy classification scheme of Benjamin (1960,1963) and Landahl (1962). Figures 7(a) and (b) respectively show the maximum disturbance growth, $G$, during the fundamental period of the forcing cycle at $R e_{\text {crit }}=3775$ (the rigid-wall value for $W o=5$ and $\Lambda=3$ ) and the Reynolds number of instability-onset as functions of the streamwise wavenumber of the disturbances. We remark that two peaks are resolved in 7 (a) and conjecture that the distinct wavenumbers at which these occur relate to the positive and negative base-flow fluctuations superimposed upon the positive mean flow within the pulsation cycle. The results arising from different levels of structural damping, $D=D_{x, s}=D_{z, s}, s=1,2$, up to its critical value are included in Figures $7(\mathrm{a})$ and (b). It can be seen that structural damping destabilizes both the transient and the timeasymptotic responses of the FSI system with a more pronounced effect on the latter. In addition, for the highest value of structural damping, the FSI system exhibits about a half order of magnitude larger maximum total growth than that of the corresponding flow through a rigid channel, which is similar to findings of figure $6(\mathrm{~b})$. We also note here that the shear-stress distribution (result not shown) is almost identical to that of the corresponding undamped compliant-wall case of figure 5(f) with structural damping generating a marginal increase to the concentration of shear-stress close to the wall.

Before closing this section, we briefly relate the present findings to human blood flow. Although the properties of the compliant walls used herein and the resulting critical Reynolds numbers are dissimilar to those in the carotid artery, the present results suggest that an increase to the stiffness and/or the damping of the arterial walls might change 
(a)

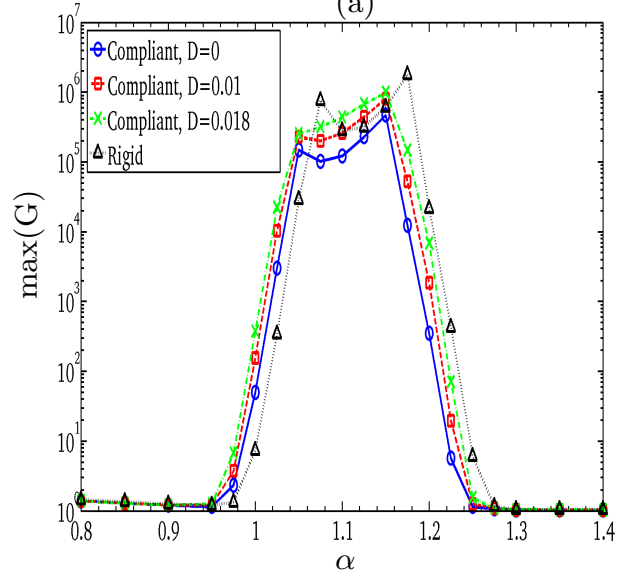

(b)

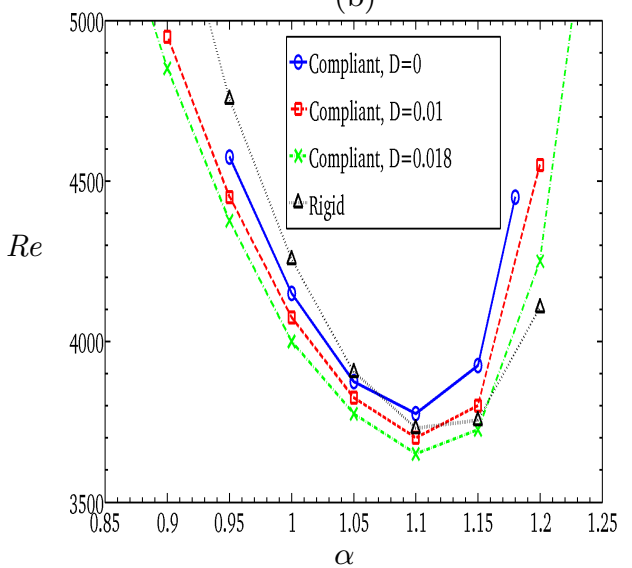

Figure 7. (a) Maximum total amplification of the disturbances, at $R e_{\text {crit. }}=3775$ and (b) Reynolds number at which the flow becomes unstable versus the streamwise wavenumber, $\alpha$, for different values of structural damping $D=D_{x, s}=D_{z, s}, s=1,2$. Parameters: $W o=5, \Lambda=3, \beta=0$, compliant-wall properties same as defined in eq.(4.1) of Davies \& Carpenter $(1997 b)$

the characteristics of the flow within the forcing cycle by causing transition from laminar to turbulent flow and thus creating the conditions for the establishment of atherosclerotic plaque. In such flows the maintenance of laminar-flow shear stress is crucial for normal vascular function and the inhibition of proliferation, thrombosis and inflammation of the vessel wall (Cunningham \& Gotlieb 2005). This echoes the clinical research of Armentano et al. (2006) who found that the arterial walls of people suffering from arterial hypertension are characterized by a lower compliance and a larger wall-material viscosity. Furthermore, Alexander (1995) reported that hypertension, that correlates with vessel stiffening, increases the risk of atherosclerosis.

\subsubsection{Modulation frequency $W_{0}=10$}

We now focus on the case with frequency modulation of the base flow at $W o=10$ using the same compliant-wall properties as when $W_{o}=5$ above. Figure 8 shows the variation of the Reynolds number at instability onset with streamwise wavenumber, $\alpha$, for both rigid and compliant channel walls and different values of modulation amplitude, $\Lambda$. The flow is destabilized by increasing $\Lambda$ but to a lesser extent than that seen for $W_{o}=5$ in figure 4 . Wall compliance is again seen to have a stabilising effect at for each $\Lambda$-case and this effect is greater than that seen in the corresponding results at $W o=5$. This is because the higher modulation frequency is better matched to the natural frequency of the compliant-wall structural dynamics.

Figure 9(a) shows the most unstable Floquet exponents of the flow over the compliant wall for the model with vertical displacements for $\Lambda=5, R e_{\text {crit. }}=5475, \alpha_{\text {crit. }}=1.0, \beta=$ 0 (the case indicated in figure 8 with the black arrow). Figure 9 (b) is a plot of the corresponding Floquet multipliers predicted by the time-stepping technique (monodromy matrix) for each of the vertical and axial-vertical displacement compliant-wall models and for different levels of structural damping (the maximum being critical damping). As in the $W o=5$ case, structural damping has a destabilizing effect on the inflectional instability mechanism. Since $\operatorname{Im}\left(k_{j}\right) \neq 0$ with $\left|k_{j}\right|>1$, the unstable solution will have frequencies both of the base flow and of the unstable solution. This can also be observed in figure $9(\mathrm{c})$, where we show the time-evolution of the disturbance velocity $u_{z}$ at the 


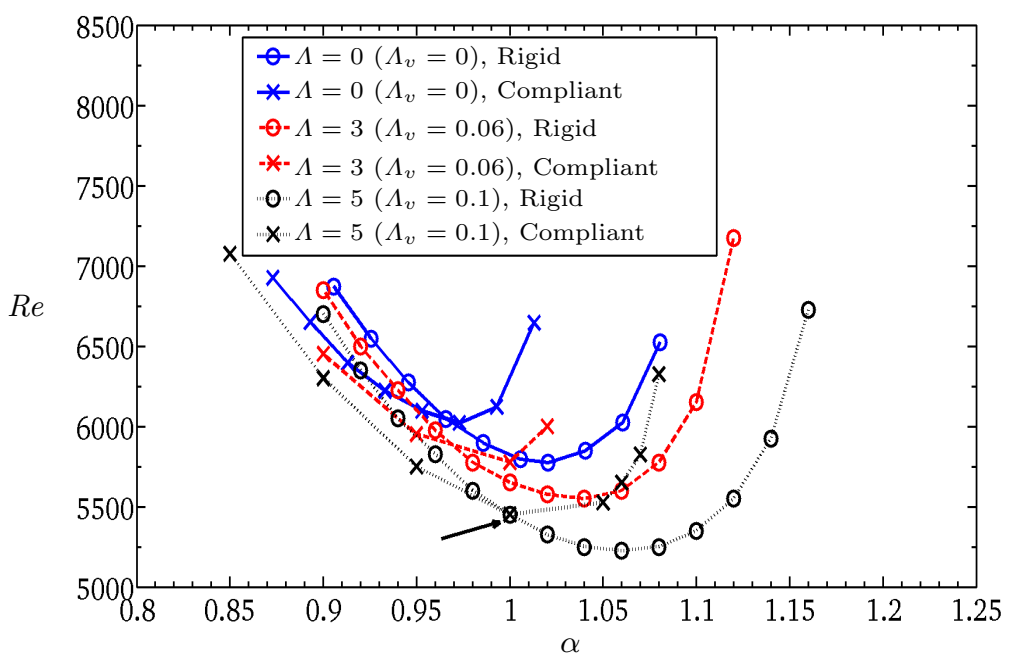

FiguRE 8. Variation of the Reynolds number at instability onset with the streamwise wavenumber $\alpha$, for rigid and compliant walls for different values of the modulation amplitude A. Parameters: $W o=10, \beta=0$, compliant-wall properties same as defined in eq.(4.1) of Davies \& Carpenter $(1997 b)$

channel center-line and on the bottom wall for the compliant-wall case. It can be seen that the relative size of the wall disturbance velocity contribution is larger than of the corresponding $W_{o}=5$ case (figure $5(\mathrm{c})$ ); this again indicates increased fluid-structure interaction between the flow-modulation and structural frequencies at this higher Wo.

In figure $9(\mathrm{~d})$, we show the growth of the disturbances, $G$, over one cycle of the pressure modulation for flow through rigid and compliant channels as well as the time-evolution of the total energy ratio $\mathrm{E}(\mathrm{t}) / \mathrm{E}(\mathrm{t}=0)$, predicted by the numerical integration with random initial conditions, for the conditions at the point marked with the black arrow in figure 8 and for different levels of structural damping. As in the $W o=5$ case, structural damping increases mildly the maximum modal transient growth, $G$ as compared with flow through a rigid channel. However, this result (and that in figure 11(e) for $\beta=0$ that is presented later), suggests that up to a pressure-modulation amplitude of $\Lambda=5$, transient effects are relatively small for $W_{o} \geqslant 10$ and that the asymptotic analysis should be adequate for the practical prediction of the system's stability with respect to the energy growth of disturbances.

\subsubsection{Modulation frequency $W_{0}=15$}

We proceed by further increasing the frequency modulation to $W o=15$ keeping the compliant-wall properties unchanged. Figure 10 shows that the flow is now stabilized by the modulation in contrast to the effect at the lower values of Wo studied in the previous subsections. This is in agreement with the work of Thomas et al. (2011), in which it was found that modulation stabilizes plane oscillatory Poiseuille flow for $W_{o} \geqslant 10 \sqrt{2}$. As found for the lower Wo cases, wall compliance continues to be stabilising but its effect is increased when a higher amplitude modulation is applied.

Figure 11(a) shows the most unstable Floquet exponents of the flow through the channel with the vertical-displacement compliant-wall model for $\Lambda=5, R e_{\text {crit }}=6500$, $\alpha_{\text {crit. }}=0.96$ and $\beta=0$ and without structural damping (the critical case indicated with the black arrow in figure 10). Figure 11(b) plots the corresponding Floquet multipliers 
(a)

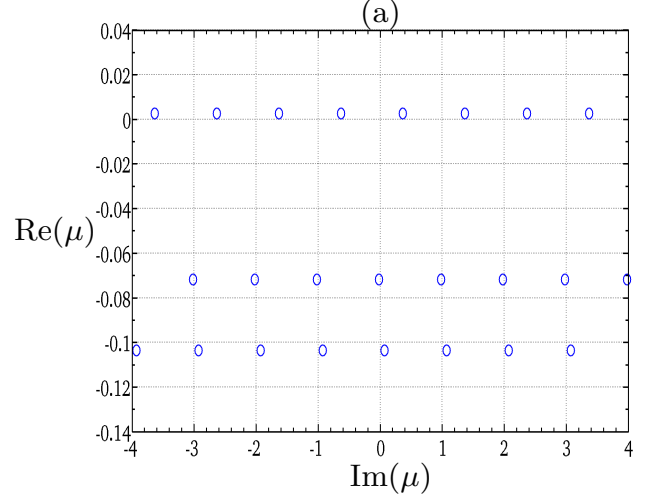

(c)

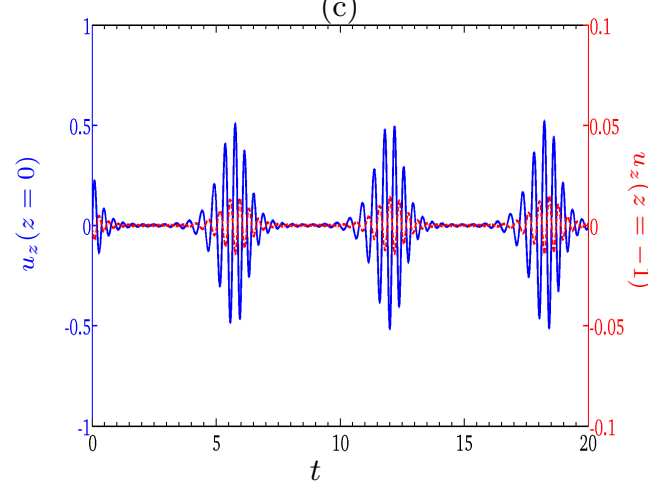

(b)

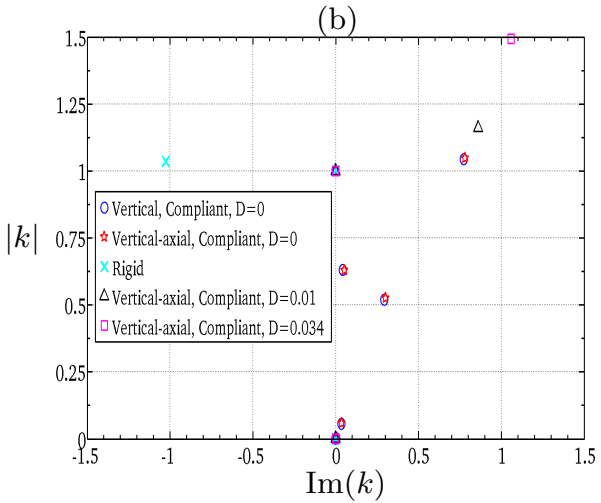

(d)



Figure 9. (a) Spectrum of the Floquet exponents for the compliant-wall case, (b) Spectrum of the Floquet multipliers for vertical and axial-vertical compliant-wall models and with different levels of structural damping, (c) Time-evolution of $u_{z}$ at the center-line of the channel (blue line) and at the bottom compliant wall (red line), (d) Growth of disturbances, $G$ and total energy ratio $\mathrm{E}(\mathrm{t}) / \mathrm{E}(\mathrm{t}=0)$ with time for compliant and rigid walls and for different values of the structural damping. Parameters: Re $e_{\text {crit. }}=5475, \alpha_{\text {crit. }}=1.0, \beta=0, W o=10, \Lambda=5$, compliant-wall properties same as defined in eq. (4.1) of Davies \& Carpenter (1997b)

predicted by the eigenvalues of the monodromy matrix for the vertical and verticalaxial structural displacements models for different levels of the structural damping (the maximum being critical damping); clearly structural damping destabilizes the timeasymptotic behaviour of the FSI system. The compliant-wall displacement velocities are larger relative to the previous cases at lower $W_{o}$ but, as seen in figure 11(d), wall compliance continues to cause only a marginal increase to the shear-stress concentration close to the wall. Finally, in figure 11(e) we show the transient growth of disturbances, $G$, and the total energy ratio $E(t) / E(t=0)$ during one cycle of the pressure modulation for different values of the structural damping, where it can be seen that the modal transient growth of the total energy of disturbances is negligible in all cases.

\subsection{Non-modal transient growth}

It should be stressed that the previous results concern modal asymptotic analysis and modal transient growth that occurs within the fundamental cycle of pulsation. However, it has been proposed by Schmid (2007) that non-modal stability theory could explain the discrepancy between the predictions of modal analysis and experimental results at pulsatile-flow frequencies relevant to blood flow in arteries. Disturbances that are predicted to be stable in the Floquet analysis could induce high levels of non-modal 


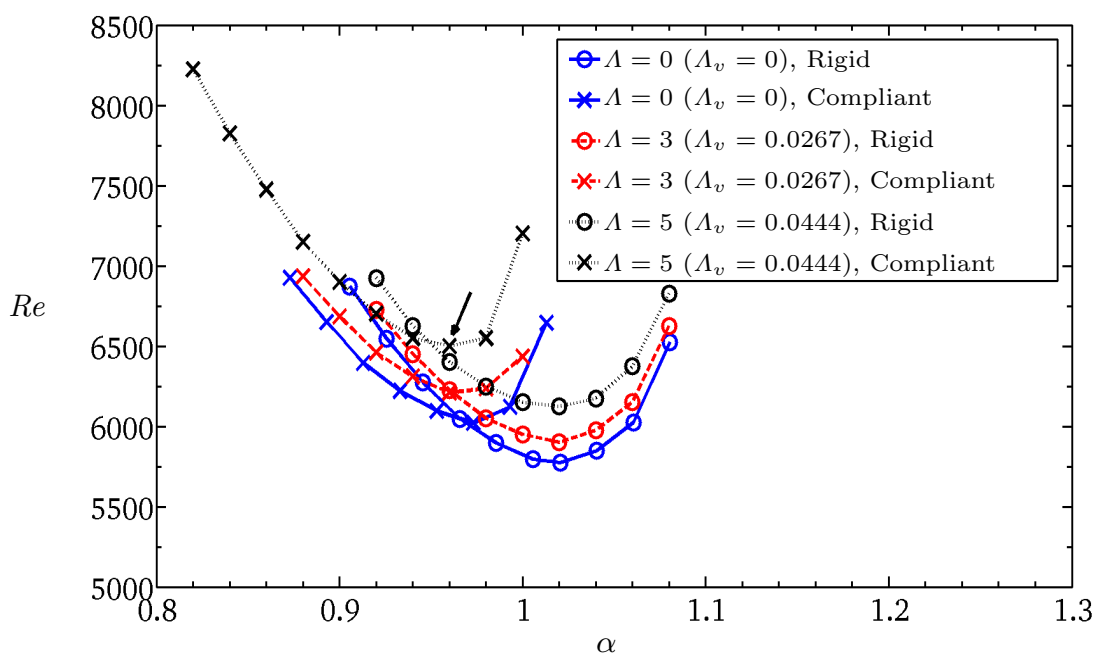

FiguRE 10. Variation of the Reynolds number at which the flow becomes unstable with the streamwise wavenumber $\alpha$, for rigid and compliant walls and for different values of the modulation amplitude $\Lambda$. Parameters: $W o=15, \beta=0$, compliant-wall properties same as defined in eq. (4.1) of Davies \& Carpenter (1997b)

transient disturbance kinetic energy downstream during the fundamental period $T$ of pulsation, or from period to period, due to the non-orthogonality of the eigenmodes of the Navier-Stokes operator. In order to assess the potential of disturbances to generate significant levels of total energy through a non-modal mechanism and compare these with those produced by modal transient growth, we define the transient growth $G_{E}$, quantified by the energy norm of the fundamental matrix $\boldsymbol{S}$, as

$$
G_{E}(t)=\max _{\hat{\boldsymbol{u}}_{z}^{0} \neq 0} \frac{\|\hat{\boldsymbol{u}}(t)\|_{E}^{2}}{\left\|\hat{\boldsymbol{u}}_{z}^{0}\right\|_{E}^{2}}=\max _{\hat{\boldsymbol{u}}_{z}^{0} \neq 0} \frac{\left\|\boldsymbol{F}(t) \exp (\boldsymbol{K} t) \hat{\boldsymbol{u}}_{z}^{0}\right\|_{2}^{2}}{\left\|\boldsymbol{F}(t) \hat{\boldsymbol{u}}_{z}^{0}\right\|_{2}^{2}}=\left\|\boldsymbol{F}(t) \exp (\boldsymbol{K} t) \boldsymbol{F}^{-1}(t)\right\|_{2}^{2},
$$

where, $\boldsymbol{G}=\boldsymbol{F}^{T} \boldsymbol{F}$ is the Gramian matrix corresponding to the total energy equation (4.11), assembled using the eigenvectors of the fundamental matrix $\boldsymbol{S}$ and $\boldsymbol{K}$, the diagonal matrix with components being its eigenvalues at each time-step. In figure 5.3(a) and (b) we plot the transient energy growth $G_{E}$ versus the time $t^{\prime}$ (with characteristic time defined through the centreline velocity, i.e. $\left.t^{\prime}=t U_{0}^{*} /\left(\omega_{f}^{*} L^{*}\right)\right)$ for different modulation amplitudes, $\Lambda$, for rigid and compliant walls when $R e=5000$ and $\alpha=1$ at each of low $\left(W_{o}=5\right)$ and high $\left(W_{o}=15\right)$ modulation frequencies.

Considering first the rigid-wall results in figure $5.3(\mathrm{a})$, it is seen that at $W_{O}=5$ the energy growth due to modal effects in pulsatile flow vastly exceeds that due to non-modal effects as can be discerned by comparing the values of the inset figure, that highlights the non-modal growth and uses a linear vertical scale, with those of the main figure 5.3 (a). Of course, when the base flow is steady $(\Lambda=0)$ only non-modal transient growth occurs; for this case excellent agreement is seen between the result of our calculation and that of Reddy et al. (1993). In contrast, figure 5.3 (b) shows that at high-frequency modulation, $W_{o}=15$, the non-modal energy-growth mechanism is stronger than that of the modal mechanism. However, at this frequency the levels of total energy extracted from the mean flow are orders of magnitude smaller those that occur for low-frequency modulation due to modal effects as seen in figure 5.3 (a) where a logarithmic scale has 
(a)

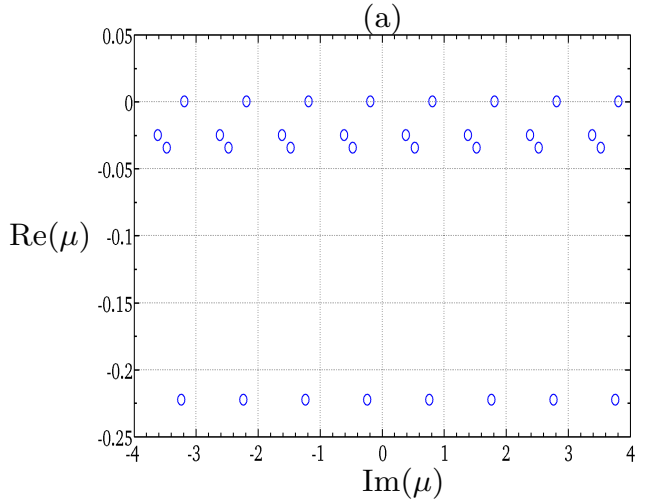

(c)

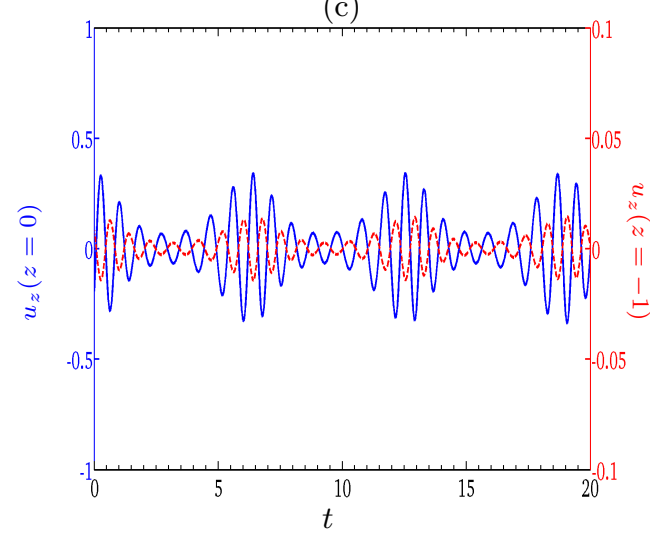

(b)

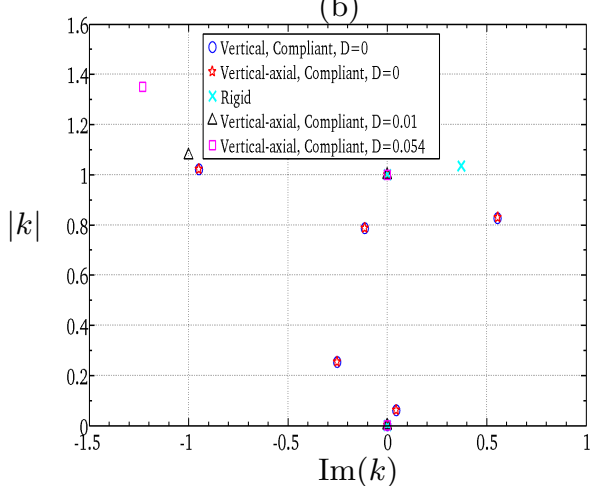

(d)

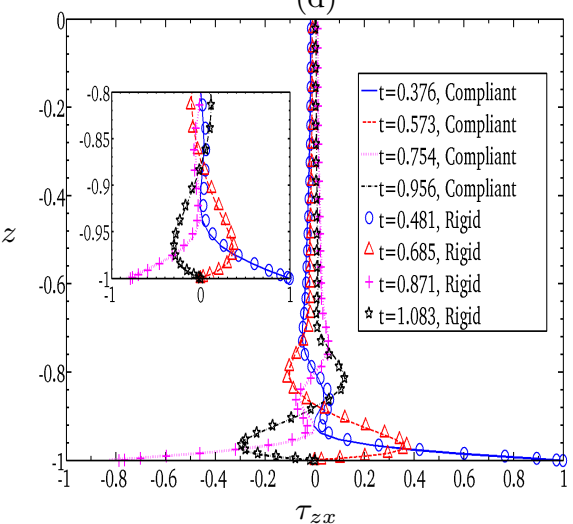

(e)



FiguRE 11. (a) Spectrum of the Floquet exponents for the compliant-wall case, (b) Spectrum of the Floquet multipliers for vertical and axial-vertical compliant-wall models and with different levels of structural damping, (c) Time-evolution of $u_{z}$ at the center-line of the channel (blue line) and at the bottom compliant wall (red line), (d) $\tau_{z x}$ distribution over one period of oscillation, (e) Growth of disturbances, $G$ and total energy ratio $E(t) / E(t=0)$ with time for compliant and rigid walls and for different values of the structural damping. Parameters: $R e_{\text {crit. }}=6500$, $\alpha_{\text {crit. }}=0.96, \beta=0, W o=15, \Lambda=5$, compliant-wall properties same as defined in eq. (4.1) of Davies \& Carpenter (1997b) 




Figure 12. Energy growth of disturbances $G_{E}\left(t^{\prime}\right)$ with time $t^{\prime}$ for different values of the modulation amplitude $\Lambda$ for rigid and compliant walls. (a) $W o=5$ and (b) $W o=15$. The dotted- and dashed-dotted lines represent the asymptotic solution of the least stable Floquet exponents, $\exp (2 \operatorname{Re}(\mu) t)$, for the rigid and compliant walls, respectively. Parameters: $R e=5000$, $\alpha=1, \beta=0$, compliant-wall properties same as defined in eq. (4.1) of Davies \& Carpenter $(1997 b)$

been used for the vertical axis. Again, we show excellent agreement between our results and those of Reddy et al. (1993). For both low- and high-frequency cases it is noted that as the modulation amplitude is increased, non-modal transient growth decreases slightly while modal transient growth increases significantly; for the low-frequency case it is seen that $\Lambda=2$ is sufficiently high that the flow is also asymptotically unstable. In addition, it can be observed from the dashed lines that the energy variation from period to period arising from modal effects closely follows the growth/decay predicted by the least stable Floquet exponents, $\exp (2 \operatorname{Re}(\mu) t)$, from the asymptotic analysis.

We now discuss the modelling and effect of wall-compliance in the transient results presented in figure 5.3. Hœpffner et al. (2010) showed that wall-compliance in steady plane Poiseuille flow increased (relative to rigid walls) the energy growth due non-modal transient growth. They obtained converged results by projecting onto the 30 least-stable eigenmodes; instability associated with the presence of spurious modes took place if this number of modes was increased excessively. This appears to be a common problem in systems, such as the present, where interfaces exist. It can be ameliorated if the order of the derivatives in the Orr-Sommerfield and the dynamic interfacial equations 
are reduced (Malik \& Hooper 2007). Accordingly, we solved for the primitive variables $u_{x}$ and $u_{z}$ explicitly and thus reduced the order of the highest derivative by one for the field and dynamic interfacial equations (2.6) and (2.8). By doing so, convergence was achieved by projecting onto the 40 least-stable eigenmodes; however a small-amplitude short-wavelength instability still persists, especially in the later stages of the modal growth; this is most prominent for high-frequency modulation. Figures 5.3(a) and (b) show that wall-compliance increases the non-modal energy growth in that the maximum of energy increases but reduces the modal transient growth for both low- and highfrequency modulation. However, due to the high value of wall stiffness used herein, the effects are fairly small. This is in qualitative agreement with the results of Hopffner et al. (2010) who showed that by increasing the wall stiffness the non-modal energy growth tended to that of the rigid-wall system for steady plane Poiseuille flow. Finally, as seen for rigid walls, figures 5.3(a) and(b) show good agreement between modal growth/decay from period to period with that predicted by the least stable Floquet exponents.

As can be seen from figures 5.3(a) and (b) the non-modal energy growth may be significant in the early stages of evolution of the initial perturbation, while at later times, it is the modal energy growth and asymptotic stability that govern the system dynamics. This is especially the case for low-frequency modulation. For high-frequency modulation the combined effects of non-modal and modal transient growth are seen to be small. We also remark that for high-frequency modulation the non-modal results for pulsatile flow should be treated with caution because the modelling effectively assumes that the timescales on which the non-orthogonal stable eigenmodes decay must be vanishingly small compared with the period of pulsation; this is exactly true for steady flow, closely adhered to for a low-frequency unsteady base flow as seen in figure 5.3(a) but is seen not to be the case for high-frequency modulation in figure 5.3(b). Overall, since the contribution of non-modal transient growth is small relative to that of modal transient growth, we will focus on the latter for the remainder of this paper. However, it is acknowledged that nonmodal transient growth can play a significant rôle for three-dimensional disturbances, for example Schmid (2007) predict that the maximum transient energy growth of oblique disturbances in rigid-walled channel flow can be up to $O\left(10^{4}\right)$, and/or for more flexible compliant walls (Hœpffner et al. 2010)

\subsection{Summary of modulation-frequency effects in plane Poiseuille flow}

We now focus on the onset of instability and therefore figure 13 summarises the variation of critical Reynolds number with the modulation frequency, Wo. It can be seen that for a given modulation amplitude there exists a range of $W_{o}$ (approximately for $W o=10$ to 30 ) where compliant-wall effects significantly stabilise the flow as evidenced by higher critical Reynolds numbers than those of the corresponding rigid channel result. Beyond this range compliance continues to have a marginally stabilising effect. It is also noted that for both rigid and compliant channels the effect of modulation is stabilising for $W_{o} \gtrsim 13$ while below this value it is strongly destabilising as compared with the stability of steady plane Poiseuille flow $(\Lambda=0$ results in the figure).

The present results indicate that as $W_{o} \rightarrow \infty$ both the rigid and compliant channel results asymptotically approach their corresponding steady base-flow critical Reynolds numbers. This might appear counter-intuitive and contradicts the findings of Singer et al. (1989) and Kerczek (1982) who showed stabilization when the modulation frequency is close to that of the frequency of neutrally stable disturbances in steady Poiseuille flow $\left(W_{o}=98.85\right)$. However, in the present work we have applied the modulation via a pressure forcing that has a defined amplitude. This means that as $W o$ is increased then the amplitude of the base-flow velocity variation is reduced; in the limit Wo $\rightarrow$ 


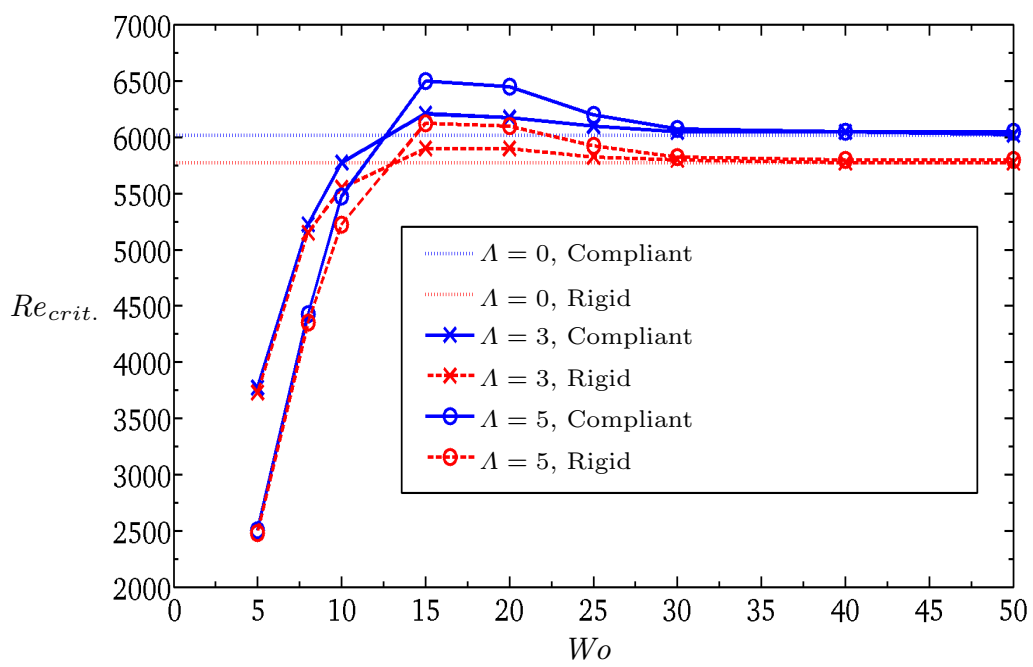

FiguRE 13. Critical Reynolds number versus $W o$, for rigid and compliant walls and for different values of the amplitude $\Lambda$ and $\beta=0$. Compliant-wall properties same as those defined in eq.(4.1) of Davies \& Carpenter (1997b)

$\infty$, the base-flow velocity variation is zero and the base flow is therefore steady. To illustrate this point of difference, Singer et al. (1989) and Kerczek (1982) used a velocitymodulation amplitude $\Lambda_{v}=0.105$ at $W_{o}=98.85$ whereas our application of a pressure modulation amplitude $\Lambda=5$ leads to $\Lambda_{v}=2 \Lambda / W o^{2}=0.001$. In most physical systems it is the imposition of a pressure modulation with finite magnitude that would generate the unsteady base flow, while the assumption of a fixed velocity modulation at very high frequencies would lead to unfeasible power requirements of the pumping system. The fact that, in the present study, the critical Reynolds numbers for both rigid and compliant channels approach those of steady Poiseuille flow provides further evidence that the inflectional instability which dominates the system behaviour at low to intermediate $W o$-values is of Class A. This is because its neutral-stability branch becomes that of the TSW branch at high Wo - that continues to exist for steady base flow - and it is well-known that TSWs are Class A instabilities.

Figure 13 also suggests that there is an additive effect from the combination of Stokes-layer and the compliant-wall stabilisation. For a given amplitude of the pressure modulation, the thickness of the Stokes layer $\delta=\left(2 \nu_{l}^{*} / \omega_{f}^{*}\right)^{1 / 2}$ reduces as the modulation frequency increases and the stabilizing effects of the Stokes layer decrease; this trend is replicated for the corresponding compliant-wall cases, albeit at higher critical Reynolds numbers, indicating that the action of the compliant walls does not modify Stokes-layer effects in pulsatile plane Poiseuille flow. Finally we report that the critical streamwise number that yields the critical Reynolds number is decreased by wall compliance, a feature seen in Figures 4, 8 and 10, while for all cases decreasing Wo leads to higher values of critical wavenumber.

\subsection{Three-dimensional disturbances in pulsatile plane Poiseuille flow}

All of the foregoing analyses have been restricted to two-dimensional disturbances, hence $\beta=0$. We now investigate the effect of oblique waves on the stability of plane pulsatile flow over compliant walls. For the relatively stiff compliant walls used in the present work, figure 14(a) shows that three-dimensional waves are asymptotically more 


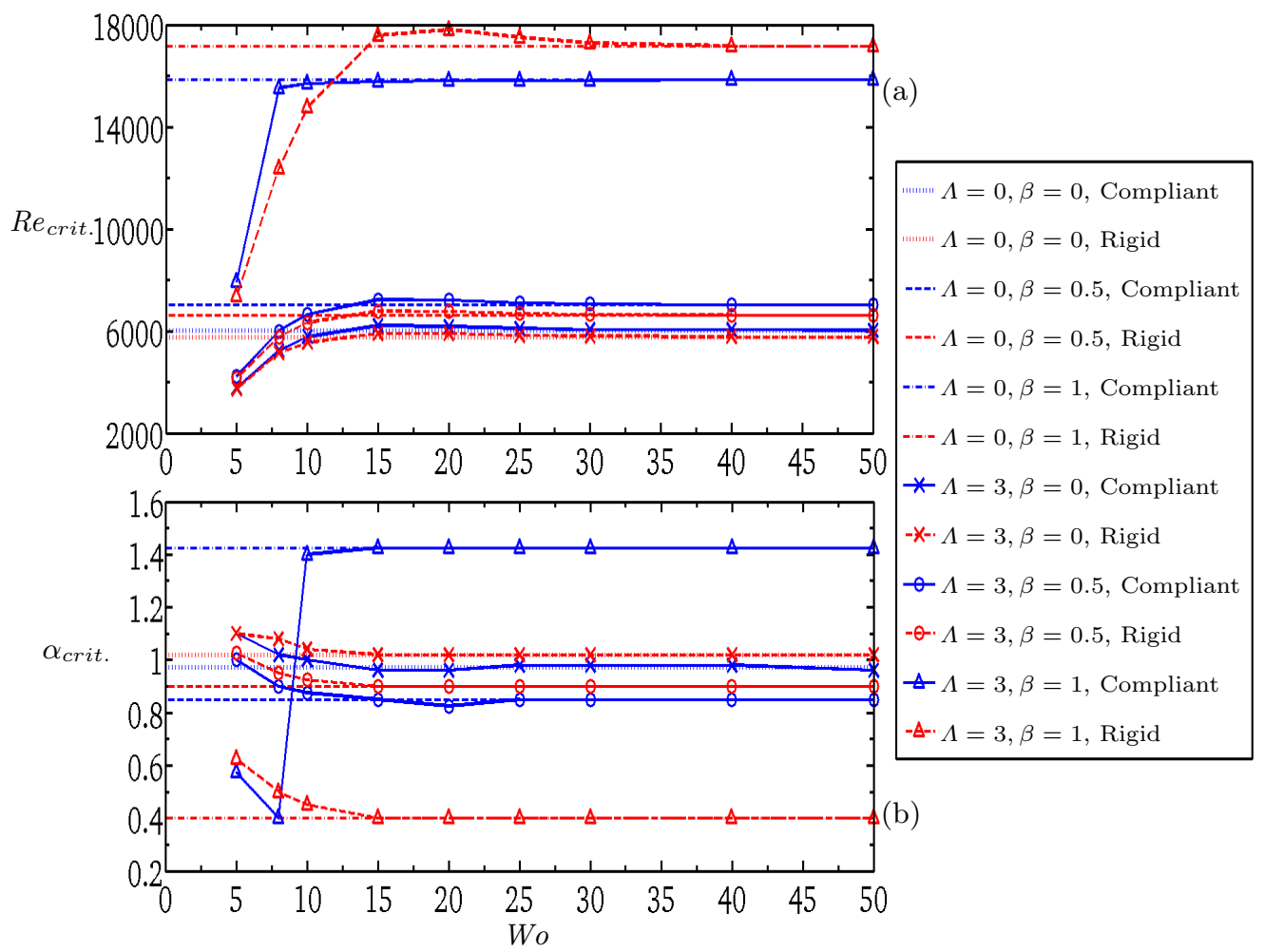

Figure 14. Variation of (a) Critical Reynolds number, and (b) critical wavenumber with Wo, for rigid and compliant walls for different values of the spanwise wavenumber, $\beta$. Compliant-wall properties same as those defined in eq. (4.1) of Davies \& Carpenter (1997b)

stable than their two-dimensional counterparts over the entire range of modulation frequencies, Wo, examined for both steady and pulsatile flow with pressure-modulation $\Lambda=3$ because their corresponding critical Reynolds numbers are higher. It is also seen in figure 14(a) that wall compliance is more effective in the stabilisation of oblique waves than for their two-dimensional counterparts. Figure 14(b) shows the variation of the streamwise wavenumber that yields the critical Reynolds number. The variations with $W o$ seen for each value of transverse wavenumber, $\beta$ for each modulation amplitude are similar to those identified in Section 5.3 as is the difference between rigid- and complaintwall systems. Overall, increasing $\beta$ decreases the critical wavenumber. However, there is one striking departure for the compliant-wall case with $\Lambda=3$ and $\beta=1$ where a jump in critical wavenumber occurs between $W_{o}=8$ and 10. This corresponds to a switch of modes and is discussed below.

Throughout this paper we have used compliant-wall properties that have been sufficiently stiff that travelling-wave flutter (TWF) has not been the critical mode for destabilisation. However, for the high Reynolds numbers associated with system destabilisation at $\Lambda=3$ and $\beta=1$, TWF replaces the inflectional instability as the critical mode when $W o>10$. This wall-based instability has much higher wavenumber as can be seen in figure 14(b). Close inspection of the critical Reynolds-number variation at $\Lambda=3, \beta=1$ in figure 14(a) reveals that this instability branch remains below the value of the critical Reynolds number for the corresponding steady base-flow result for all $W_{o}>10$ and significantly lower than the critical Reynolds number for the rigid-walled channel (in 
which TWF cannot exist). For the TWF instability, there is no range of $W o$ for which base-flow pulsation is stabilizing and this therefore suggests that the Stokes layer is a further destabilising source of irreversible energy transfer from the flow to the wall.

TWF is a Class B instability and for this reason is stabilized by structural damping. This is seen in figure 15(a), that shows the variation of Reynolds number at instability onset with streamwise wavenumber with different amounts of structural damping for the case with $W_{o}=15, \Lambda=3$ and $\beta=1$. Clearly there exist two types of instability branches: the rigid and compliant channel branch at lower wavenumbers associated with inflectional instability while the second, at higher wavenumbers, for the compliant channel is that of TWF. For an elastic wall, the TWF branch yields a critical Reynolds number lower than that of the rigid-walled system and this continues to hold for an incremental level of damping that is seen to be stabilising. However, when a sufficiently high level of structural damping is present, the critical Reynolds number of TWF exceeds that of the inflectional instability of the pulsatile flow through a rigid channel. At the same time, the structural damping destabilizes the inflectional instability branch of the compliant channel and it may become the critical instability.

While the structure of neutral-stability curves in figure 15(a) parallels that found in Davies \& Carpenter (1997a) for steady flow through a compliant channel using a two-dimensional analysis $(\beta=0)$ wherein TSWs and TWF are the principal system instabilities, the static divergence instability that they also found at low wavenumbers is absent in our results. We remark that for their figure 7, Davies \& Carpenter (1997a) used a tensioned membrane instead of a spring-backed plate and this resulted in a very flexible channel that is more prone to wall-based instabilities. For the spring-backed flexible plate used in our system, an equivalent critical Reynolds number predicted for the onset of two-dimensional divergence instability for steady inviscid fluid flow in a compliant channel (Burke et al. 2014) is $R e_{D} \approx 1.25 \times 10^{4}$, having chosen blood for the fluid and characteristic dimension typical of a small blood vessel. This value falls within the range of Reynolds numbers in figure 15(a). However, potential flow is known to yield a very low estimate of divergence-onset flow speed (equivalent Reynolds number) because it does not account for the effects of flow shear (Davies 2003; Kapor \& Lucey 2012). In addition, it has been shown by Lucey \& Carpenter (1993) that two-dimensional disturbances give significantly lower values of divergence-onset flow speed than their three-dimensional counterparts. The combination of these effects accounts for the fact that we do not find divergence eigenmodes in the range of Reynolds numbers used to generate figure 15(a).

In closing this subsection, we emphasise that for the present parameters, the stability of the system will be determined by the inflectional instability of plane waves with $\beta=0$. Nevertheless, for compliant channels with more flexible walls than those used in the present paper, the existence of TWF or divergence may determine the stability bounds for certain combinations of system parameters. Finally, a transient modal analysis yielding the results presented in figure 15(b) predicts that the maximum amplification of the total energy of system disturbances, $G$, reduces as the wavelength of the spanwise disturbances decreases for both rigid and compliant channels.

\section{Conclusions}

The time asymptotic (Floquet) stability and modal transient growth during the pressure-modulation cycle of pulsatile plane Poiseuille flow bounded by compliant walls has been investigated, implementing both vertical and vertical-axial displacement models for the compliant walls. Two equivalent numerical methods were developed for the stability analysis, namely a matrix method based on the decomposition of the 
(a)

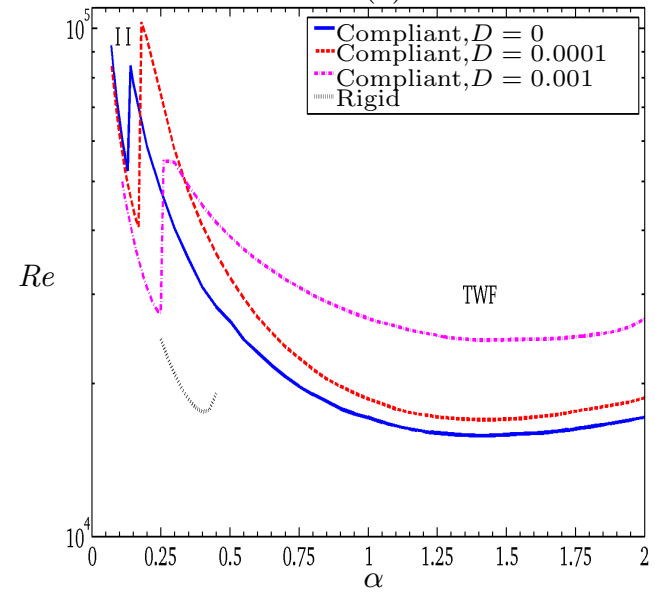

(b)

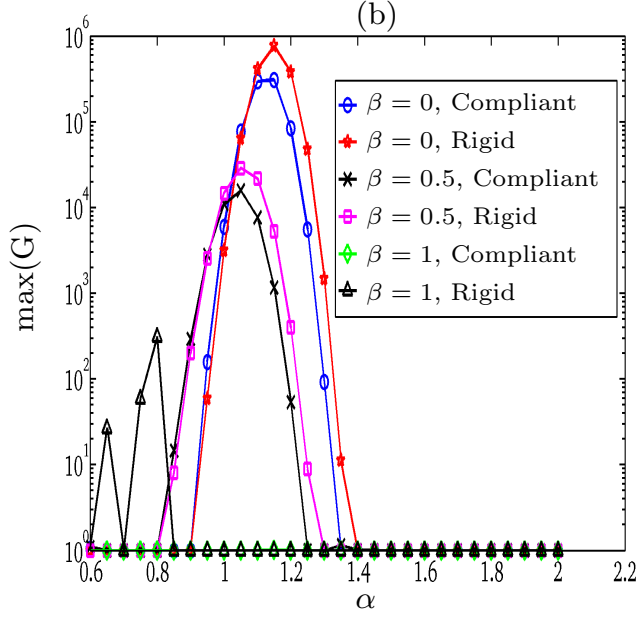

FiguRE 15. (a) Variation of Reynolds number at which the flow becomes unstable with the streamwise wavenumber for different levels of structural damping for the compliant-wall case when $W_{o}=15, \Lambda=3$ and $\beta=1$. II and TWF denote Inflectional and TWF instability branches, respectively. (b) Maximum amplification of disturbances for the case with $W o=5, \Lambda=3, R e_{\text {crit. }}=3775$ against the streamwise wavenumber, $\alpha$, for different transverse wavenumbers, $\beta$. Compliant wall properties same as those defined in eq.(4.1) of Davies \& Carpenter $(1997 b)$

unknown amplitude of the disturbance into a product of exponential growth and sum of harmonics and a time-stepping technique based on the temporal evolution of the fundamental solution matrix. In addition, transient growth due to non-normality of the Navier-Stokes operator has been quantified and contrasted with that due to modal transient growth.

For the relatively stiff compliant walls used in the present study, the pulsatile flow is always stabilized by the flexibility of the compliant walls for the whole range of Womersley numbers and amplitudes of the pressure modulation examined. This effect combines favourably with the stabilizing effect of the Stokes layer in pulsatile flows. Structural damping in the compliant walls has a destabilizing effect on the inflectional (Rayleigh) instability that dominates the stability of pulsatile plane Poiseuille flow.

It was shown that modal transient growth within the period of pulsation may be very significant when $W_{o}<10$ for the amplitudes of pressure modulation used in the present study even when the flow is asymptotically stable in that its eigenmodes are exponentially decaying. The flexibility of the complaint wall serves to reduce this modal transient growth; this effect is small for the relatively stiff complaint walls used in the present study. In contrast, non-modal transient growth when $W_{o}<10$ is shown to be very small (as compared to that generated by the modal mechanism) and its maximum is increased (relative to that for a rigid wall) by wall compliance. The differences in stability bounds between the vertical and vertical-axial compliant-wall displacement models were insignificant for the relatively high Reynolds numbers used in the present study.

For the properties of the compliant walls used herein, three-dimensional waves are more stable than their two-dimensional counterparts and wall compliance is more effective in their stabilisation because they tend to become unstable at higher Reynolds numbers than those of two-dimensional waves. For system parameters that lead to high Reynolds numbers for destabilisation through the inflectional instability, wall-based travelling-wave flutter (TWF) can become the critical mode. In contrast to the inflectional instability, 
TWF is stabilized by structural stiffness and damping and destabilized by the Stokes layer. It is noted that very compliant channels may lead to TWF featuring more prominently at the lower Reynolds numbers associated with inflectional instability for plane waves. Finally, the maximum amplification of three-dimensional disturbances within the pressure-modulation cycle is found to be lower than that of their two-dimensional counterparts.

The Floquet stability analysis with the wave-type approximation for the streamwise and transverse directions conducted in the present study does not take into account the structural boundary conditions of a finite-length compliant section in the channel. It has recently been shown by Tsigklifis \& Lucey (2015), studying the global stability of Blasius boundary-layer flow over a compliant panel, that flow-based Tollmien-Schlichting waves (TSW) and the wall-based TWF instabilities can resonate with eigenfrequencies of a finite-length compliant wall to create global instabilities. The time-stepping method introduced in the present work could serve as the platform for the extension of the present analyses to include spatial non-uniformity of disturbances in the streamwise and/or the spanwise direction, thus taking into account the structural boundary conditions of a finite-length FSI system conveying pulsatile flow.

\section{REFERENCES}

Alexander, R. W. 1995 Hypertension and the pathogenesis of atherosclerosis. oxidative stress and the mediation of arterial inflammatory response: A new perspective. Hypertension 25, 155-161.

Armentano, R. L., Barra, J. G., Santana, D. B., Pessana, F. M., Graf, S., Craiem, D., Brandani, L. M., Baglivo, H. P. \& Sanchez, R. A. 2006 Smart damping modulation of carotid wall energetics in human hypertension: Effects of angiotensin-converting enzyme inhibition. Hypertension 47, 384-390.

Benjamin, T. B. 1960 Effects of a flexible boundary on hydrodynamic stability. Journal of Fluid Mechanics 9, 513-532.

Benjamin, T. B. 1963 The three-fold classification of unstable disturbances in flexible surfaces bounding inviscid flows. Journal of Fluid Mechanics 16, 436-450.

Burke, M. A., Lucey, A. D., Howell, R. M. \& Elliott, N. S.J. 2014 Stability of a flexible insert in one wall of an inviscid channel flow. Journal of Fluids and Structures 48, 435 450.

Canuto, C., Hussaini, M.Y., Quarteroni, A. \& Zang, T.A. 1988 Methods in Fluid Dynamics. Springer-Verlag.

Carpenter, P. W. \& GajJar, J. S. B. 1990 A general theory for two and three-dimensional wall-mode instabilities in boundary layers over isotropic and anisotropic compliant walls. Journal of Theoretical and Computational Fluid Dynamics 1, 349-378.

Carpenter, P. W. \& Morris, P. J. 1990 The effects of anisotropic wall compliance on boundary-layer stability and transition. Journal of Fluid Mechanics 218, 171-223.

Cooper, A. J. \& Carpenter, P. W. 1997 The stability of rotating-disc boundary-layer flow over a compliant wall. part 1. type i and ii instabilities. Journal of Fluid Mechanics 350, $231-259$.

Cunningham, K. S. \& Gotlieb, A. I. 2005 The role of shear stress in the pathogenesis of atherosclerosis. Laboratory Investigation 85, 9 - 23.

Davies, C. 2003 Convective and absolute instabilities of flow over compliant walls. In Flow Past Highly Compliant Boundaries and in Collapsible Tubes, , vol. 72, pp. 69-93. Springer Netherlands.

Davies, C. \& Carpenter, P. W. $1997 a$ Instabilities in a plane channel flow between compliant walls. Journal of Fluid Mechanics 352, 205-243.

Davies, C. \& CARpenter, P. W. $1997 b$ Numerical simulation of the evolution of TollmienSchlichting waves over finite compliant panels. Journal of Fluid Mechanics 335, 361-392. 
Davis, S. H. 1976 The stability of time-periodic flows. Annual Review of Fluid Mechanics 8, $57-74$.

EinaV, S. \& Sokolov, M. 1993 An experimental study of pulsatile pipe flow in the transition range. Journal of Biomechanical Engineering 115, 404-411.

Gaster, M. 1988 Is the dolphin a red herring? In Turbulence Management and Relaminarisation, pp. 285-304. Springer-Berlin Heidelberg.

HAll, P. 1975 The stability of Poiseuille flow modulated at high frequencies. Proceedings of the Royal Society A 344, 453-464.

Hepffiner, J., Bottaro, A. \& Favier, J. 2010 Mechanisms of non-modal energy amplification in channel flow between compliant walls. Journal of Fluid Mechanics 642, 489 - 507.

Iooss, G. \& JosePH, D. D. 1990 Elementary Stability and Bifurcation Theory. Springer-Verlag New York.

KAPOR, J. S. \& LuCEY, A. D. 2012 Flow-induced vibrations of a flexible panel in a boundarylayer flow. In Flow-Induced Vibration, 10th International Conference on Flow-Induced Vibration (E Flow-Induced Noise) FIV2012, pp. 647 - 654.

Kapor, J. S., Lucey, A. D. \& Pitman, M. W. 2009 Boundary-layer hydrodynamics using mesh-free modelling. In The 18th World IMACS Congress and MODSIM09 International Congress on Modelling and Simulation.

Kerczek, C. H. Von 1982 The instability of oscillatory plane Poiseuille flow. Journal of Fluid Mechanics 116, 91-114.

Kerczek, C. V. \& DAvis, S. H. 1974 Linear stability theory of oscillatory Stokes layers. Journal of Fluid Mechanics 62, 753-773.

LANDAHL, M. T. 1962 On the stability of a laminar incompressible boundary layer over a flexible surface. Journal of Fluid Mechanics 13, 609-632.

Lucey, A. D. \& CARPenter, P. W. 1992 A numerical simulation of the interaction of a compliant wall and inviscid flow. Journal of Fluid Mechanics 234, 121-146.

Lucey, A. D. \& CARPenter, P. W. 1993 The hydroelastic stability of three-dimensional disturbances of a finite compliant wall. Journal of Sound and Vibration 165 (3), 527-552.

Lucey, A. D. \& Carpenter, P. W. 1995 Boundary layer instability over compliant walls: Comparison between theory and experiment. Physics of Fluids 7, 2355-2363.

Malik, S. V. \& Hooper, A. P. 2007 Three-dimensional disturbances in channel flows. Physics of fluids 19, 052102-1 - 052102-18.

Mao, X., Sherwin, S. J. \& Blackburn, H. M. 2009 Transient growth and bypass transition in stenotic flow with a physiological waveform. Theoretical and Computational Fluid Dynamics 25, 31-42.

MeYer, C. D. 2000 Matrix Analysis and Applied Linear Algebra. SIAM New York.

Nerem, R. M., Seed, W. A. \& Wood, N. B. 1972 An experimental study of the velocity distribution and transition to turbulence in the aorta. Journal of Fluid Mechanics 52, $137-160$.

Pitman, M. W. \& Lucey, A. D. 2009 On the direct determination of the eigenmodes of finite flow-structure systems. Proceedings of the Royal Society A 465, 257-281.

Reddy, S. C., Schmid, P. J. \& Henningson, D. S. 1993 Pseudospectra of the Orr-Sommerfeld operator. SIAM Journal on Applied Mathematics $\mathbf{5 3}$ (1).

Schmid, P. J. 2007 Nonmodal stability theory. Annual Review of Fluid Mechanics 39, 129-162.

Schmid, P. J. \& Henningson, D. S. 2001 Stability and Transition in Shear Flows. SpringerVerlag New York.

Schmid, P. J. \& KytomaA, H. K. 1994 Transient and asymptotic stability of granular shear flow. Journal of Fluid Mechanics 264, 255-275.

Seydel, R. 1988 From Equilibrium to Chaos. Practical Bifurcation and Stability Analysis. Elsevier, New York.

Shankar, V. \& Kumaran, V. 2002 Stability of wall modes in fluid flow past a flexible surface. Physics of fluids 14, 2324-2338.

Singer, B. A., Ferziger, J. H. \& Reed, H. L. 1989 Numerical simulations of transition in oscillatory plane channel flow. Journal of Fluid Mechanics 208, 45-66.

Stratman, A. G., Khayat, R. E., Haj-Qhasem, E. \& Steinman, D. A. 2002 On the hydrodynamic stability of pulsatile flow in a plane channel. Physics of Fluids 14, 19381944 . 
Thaokar, R. M. \& Kumaran, V. 2004 Stability of oscillatory flows past compliant surfaces. The European Physical Journal B 41, 135-145.

Thomas, C., Bassom, A. P., Blennerhassett, P. J. \& Davies, C. 2011 The linear stability of oscillatory Poiseuille flow in channels and pipes. Proceedings of the Royal Society A 467, 2643-2662.

Timoshenko, S. \& Woinowsky-Krieger, S. 1959 Theory of plates and shells. McGraw-Hill.

Tsigklifis, K. \& LuceY, A. D. 2015 Global instabilities and transient growth in Blasius boundary-layer flow over a compliant panel. Sadhana 40, 945-960.

Tsiglifis, K. \& Pelekasis, N. A. 2011 Parametric stability and dynamic buckling of an encapsulated microbubble subject to acoustic disturbances. Physics of fluids 23, 012102$1-012102-28$.

YEO, K. S. 1992 The three-dimensional stability of boundary-layer flow over compliant walls. Journal of Fluid Mechanics 238, 537-577. 